\title{
In vitro reconstitution of yeast splicing with U4 snRNA reveals multiple roles for the $3^{\prime}$ stem-loop
}

\author{
AMY J. HAYDUK, ${ }^{1}$ MARTHA R. STARK, ${ }^{1}$ and STEPHEN D. RADER ${ }^{2}$ \\ Department of Chemistry, University of Northern British Columbia, Prince George, British Columbia, V2N 4Z9 Canada
}

\begin{abstract}
U4 small nuclear RNA (snRNA) plays a fundamental role in the process of premessenger RNA splicing, yet many questions remain regarding the location, interactions, and roles of its functional domains. To address some of these questions, we developed the first in vitro reconstitution system for yeast $\mathrm{U} 4$ small nuclear ribonucleoproteins (snRNPs). We used this system to examine the functional domains of $U 4$ by measuring reconstitution of splicing, U4/U6 base-pairing, and triple-snRNP formation. In contrast to previous work in human extracts and Xenopus oocytes, we found that the $3^{\prime}$ stem-loop of U4 is necessary for efficient base-pairing with U6. In particular, the loop is sensitive to changes in both length and sequence. Intriguingly, a number of mutations that we tested resulted in more stable interactions with U6 than wild-type U4. Nevertheless, each of these mutants was impaired in its ability to support splicing, indicating that these regions of U4 have functions subsequent to base pair formation with U6. Our data suggest that one such function is likely to be in tri-snRNP formation, when U5 joins the U4/U6 di-snRNP. We have identified two regions, the upper stem of the $3^{\prime}$ stem-loop and the central domain, that promote tri-snRNP formation. In addition, the loop of the $3^{\prime}$ stem-loop promotes di-snRNP formation, while the central domain and the $3^{\prime}$-terminal domain appear to antagonize di-snRNP formation.
\end{abstract}

Keywords: pre-mRNA splicing; in vitro reconstitution; U4 snRNA; di-snRNP formation; spliceosome activation; protein-RNA interaction; splicing complementation

\section{INTRODUCTION}

Splicing, an essential step in eukaryotic gene expression, involves the removal of noncoding introns from precursor messenger RNA (pre-mRNA) and ligation of the flanking protein-coding exons to produce mature mRNA. This process is catalyzed by a large ribonucleoprotein complex, the spliceosome, composed of five small nuclear RNAs (snRNAs)-U1, U2, U4, U5, and U6-and over 100 proteins, some of which associate with a specific snRNA to form the corresponding small nuclear ribonucleoprotein (snRNP). Through a highly ordered and tightly regulated series of interactions, these snRNPs assemble onto the pre-mRNA and, following a number of conformational changes leading to formation of the active spliceosome, catalyze the two chemical steps of splicing.

While each of the five snRNAs plays an essential role in splicing, that of U4 remains the least well understood. U4

\footnotetext{
${ }^{1}$ These authors contributed equally to this work.

${ }^{2}$ Corresponding author.

E-mail rader@unbc.ca.

Article published online ahead of print. Article and publication date are at http://www.rnajournal.org/cgi/doi/10.1261/rna.031757.111.
}

and U6 share extensive sequence complementarity (Brow and Guthrie 1988; Guthrie and Patterson 1988), and their interaction in the form of a di-snRNP is required for incorporation of U6 into the active spliceosome (Brow and Guthrie 1988). U4, however, is not directly involved in splicing catalysis and is thought to dissociate from the spliceosome prior to the first chemical reaction (Lamond et al. 1988; Yean and Lin 1991). Furthermore, there has been no direct biochemical identification of the proteins associated with U4 snRNA in the free snRNP, the sequences with which they interact, or the roles they may perform during snRNP and spliceosome assembly.

A powerful tool for examining the function and interactions of the U4 snRNP is in vitro reconstitution. This involves depleting endogenous U4 snRNA from cell extract to abolish splicing activity, followed by complementation with a modified version of U4 to examine the effects of mutations or investigate its interaction partners. Because U4 function is essential, in vitro reconstitution enables analysis of lethal mutations that would not be possible using in vivo methods. In vitro reconstitution assays have been developed for all five mammalian snRNPs (Wersig et al. 1992; Wolff and Bindereif 1992; Ségault et al. 1995; Will et al. 1996), as well as yeast (Saccharomyces cerevisiae) U2, U5, and U6 (Fabrizio et al. 
1989; McPheeters et al. 1989; O’Keefe et al. 1996); however, there has been no report of in vitro reconstitution of yeast $\mathrm{U} 1$ or U4, though the latter has been attempted (Horowitz and Abelson 1993).

Reconstitution of mammalian U4 has provided valuable insight into the functionally important regions of this molecule. U4 snRNA can be divided into six domains: stem II, the $5^{\prime}$ stem-loop, stem I, the central domain, the $3^{\prime}$ stem-loop (the central stem-loop in mammalian $\mathrm{U} 4$ ), and the $3^{\prime}$ terminal domain containing the Sm binding site (Fig. 1A). By examining deletion mutations within each of these domains, Wersig and Bindereif (1990, 1992) demonstrated that the $5^{\prime}$ portion of U4, including stems I and II and the intervening $5^{\prime}$ stem-loop, is required for U4/U6 di-snRNP formation, subsequent spliceosome assembly, and splicing in vitro, while the $3^{\prime}$ portion of the molecule appeared to be dispensable.

To investigate the domain structure and interactions of yeast U4, we have developed an assay for in vitro reconstitution of functional U4 snRNPs. By using DNA oligonucleotide-targeted degradation by RNase $\mathrm{H}$, extract was specifically depleted of endogenous U4, abolishing splicing. Subsequent addition of in vitro transcribed (IVT) U4 snRNA restored up to $50 \%$ of the splicing activity of the extract. By using this system, we were able to define the minimum functional sequence of yeast U4 snRNA. Our results reveal that in contrast to mammalian U4, the $3^{\prime}$ stem-loop of yeast $\mathrm{U} 4$ is essential for interaction with U6 and thus for splicing. Strikingly, a number of U4 mutants base-paired to U6 more efficiently than wild-type U4. The observation that these mutants were nevertheless impaired in their ability to support splicing demonstrates that U4 plays an important role subsequent to U4/U6 di-snRNP formation.
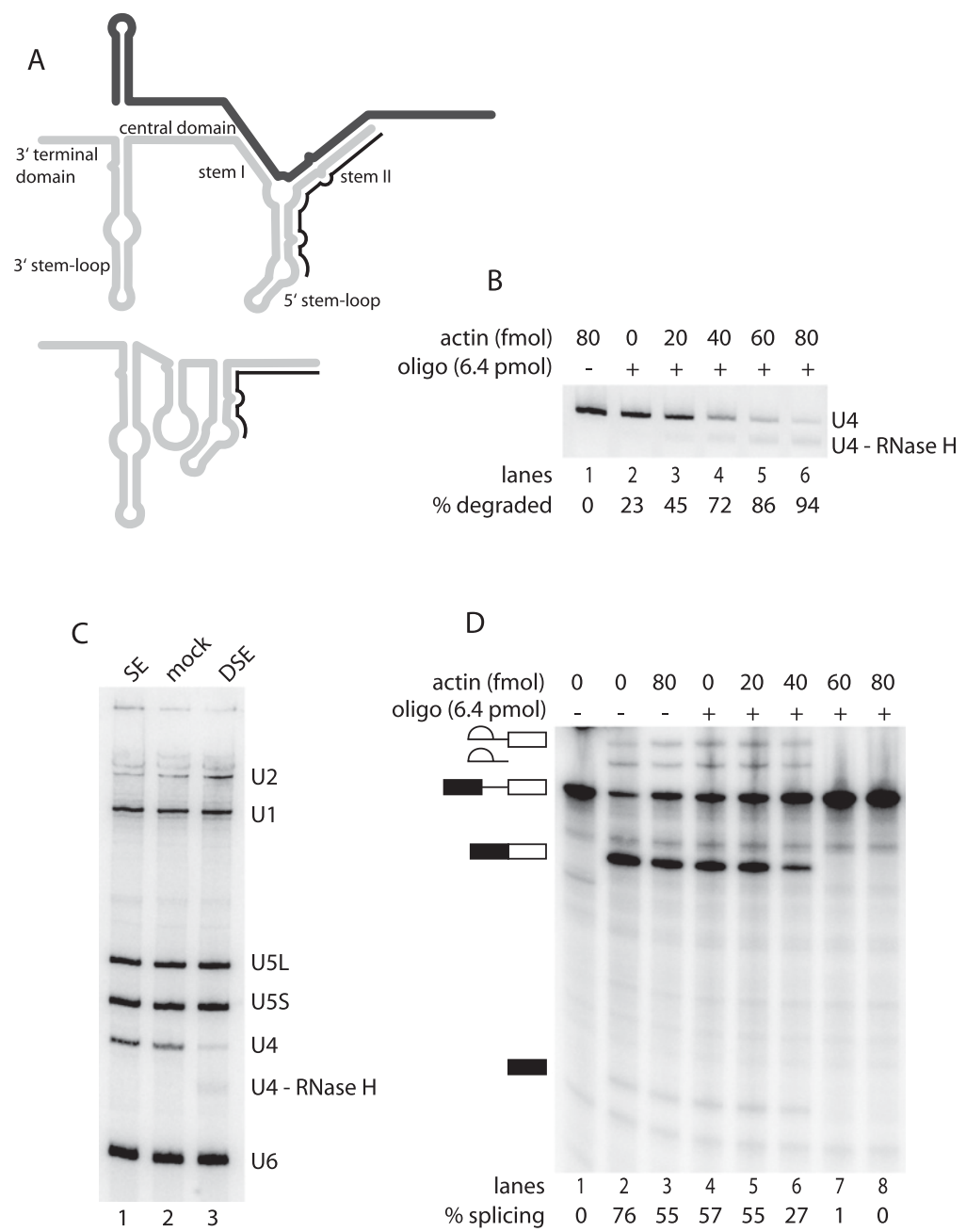

D

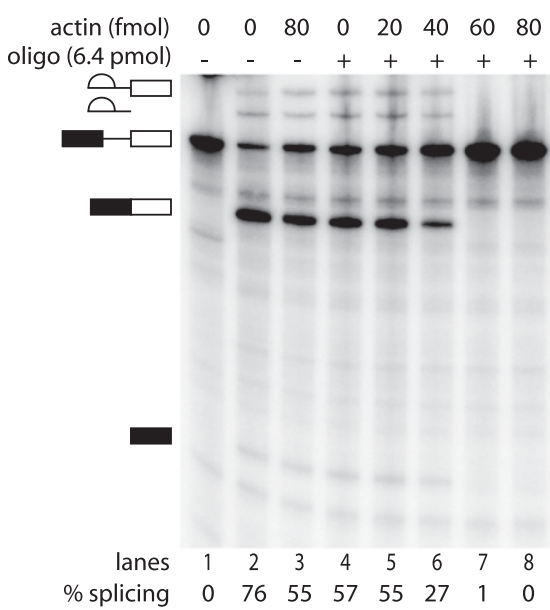

FIGURE 1. U4 degradation and consequent splicing inhibition require active splicing conditions. (A) Secondary structure of U4 (light gray) base-paired to U6 (dark gray) (Brow and Guthrie 1988) and as a free particle (Myslinski and Branlant 1991), with the targeting oligonucleotide binding site indicated (black line). (B) Extent of U4 degradation increases with actin concentration. Northern blot of U4 in splicing extract in the absence or presence of targeting oligonucleotide and increasing amounts of unlabeled actin pre-mRNA. (C) U4 degradation does not affect other snRNAs. Denaturing Northern blot of untreated splicing extract (SE, lane 1), mock-depleted extract (lane 2), and U4 depleted extract (DSE, lane 3), probed for all five yeast snRNAs as indicated on the side. U5L indicates long U5; U5S, short U5; and U4-RNaseH, U4 cleavage product. (D) Extent of splicing inhibition increases with the concentration of cold actin in the U4 degradation step. Denaturing gel showing the generation of splicing intermediates and products after incubation of radioactively labeled actin premRNA in mock-depleted (lane 3) or U4-depleted (lanes 4-8) extract preincubated with increasing amounts of unlabeled actin pre-mRNA. Lanes 1 and 2 are standard splicing controls incubated for 0 or $30 \mathrm{~min}$, respectively. Lanes $3-8$ are the same reactions as in panel $B$. Bands corresponding to splicing substrate, intermediates, and products are indicated to the left of the gel (black and white boxes, $5^{\prime}$ and $3^{\prime}$ exons; black line, intron). The absolute splicing efficiency of each reaction is indicated below the gel.

\section{RESULTS}

\section{The $5^{\prime}$ end of $U 4$ snRNA is efficiently degraded under conditions of active splicing}

To develop an in vitro assay for reconstitution of functional yeast U4 snRNPs, we needed to inactivate the endogenous
U4 and thereby block the splicing activity of the extract. An effective technique for depletion of specific RNA molecules from extract is oligonucleotide-targeted degradation by RNase H. A previous attempt to develop a yeast U4 in vitro reconstitution assay used a complementary DNA oligonucleotide to direct RNase $\mathrm{H}$ degradation of $\mathrm{U} 4$ nucleotides 72-92 but was unsuccessful (Horowitz and 
Abelson 1993). This oligonucleotide had been shown to direct nearly complete degradation of the target sequence (Xu et al. 1990); however, degradation of this central region of U4 failed to abolish splicing activity (Horowitz and Abelson 1993), presumably because a functional portion of U4 remained intact. Here, we instead targeted the $5^{\prime}$ end of U4 (Fig. 1A), as it has been shown to be essential for interaction with U6 and, consequently, for splicing (Vankan et al. 1990; Wersig and Bindereif 1990, 1992).

Normally, the $5^{\prime}$ end of U4 is found base-paired to U6, forming intermolecular stem II (Fig. 1A), and, as a result, is almost completely resistant to oligonucleotide-targeted RNase $\mathrm{H}$ degradation (Xu et al. 1990). To circumvent the protection provided by U4/U6 stem II and to accomplish efficient U4 degradation, we took advantage of the changes in the $\mathrm{U} 4$ secondary structure that take place during the splicing cycle. For the active spliceosome to be formed, basepairing interactions between U4 and U6 must be broken, resulting in the release of $\mathrm{U} 4$ from the spliceosome in the form of a free particle. In this U4 species, the $5^{\prime}$ region normally engaged in base-pairing with U6 is believed to adopt a largely single-stranded conformation that should be more accessible to the targeting DNA oligonucleotide (Fig. 1A). To produce conditions of active splicing, unlabeled actin pre-mRNA was added to yeast splicing extract in the presence of ATP. Titration of actin pre-mRNA showed that increasing amounts of pre-mRNA allowed higher U4 degradation efficiencies, with $80 \mathrm{fmol}$ pre-mRNA resulting in nearly complete depletion of full-length U4 (Fig. 1B). The amount of actin pre-mRNA necessary for efficient depletion of U4 was found to be extract-dependent, ranging from 15-80 fmol. Under these conditions, U4 snRNA is specifically depleted, and there is no effect on the other snRNAs (Fig. 1C). The remaining U4 fragment (nucleotides [nt] 31-160, labeled U4-RNaseH) is visible but is substantially degraded.

\section{Degradation of the 5' end of U4 snRNA inhibits splicing in vitro}

A critical requirement for the development of a useful U4 in vitro reconstitution assay is that degradation of endogenous U4 must inhibit the splicing activity of the extract. To test the effectiveness of splicing inhibition, U4-depleted extract was incubated with radioactively labeled pre-mRNA, followed by gel electrophoresis to separate the splicing substrate from any intermediates or products formed. Near-complete depletion of the full-length U4 resulted in complete inhibition of splicing in the extract relative to the mock-depleted control (Fig. 1D, cf. lanes 3 and 8). Given that the number of splicing cycles that can be performed by a specific extract is limited (Raghunathan and Guthrie 1998a), it was important to ensure that the splicing performed during the degradation of U4 was not compromising the splicing potential of the extract. Incubation of mock-depleted extract in the presence of $80 \mathrm{fmol}$ pre-mRNA decreased the subsequent splicing activity of the extract by $\sim 20 \%$ compared with mock-depleted extract incubated in the absence of pre-mRNA (cf. lanes 2 and 3). Mock-depleted extract was generally able to splice $\sim 40 \%-70 \%$ of the labeled actin pre-mRNA, while depletion of $\mathrm{U} 4$ consistently reduced this number to $<1 \%$.

\section{IVT U4 RNA restores splicing in U4-depleted extract}

Having identified conditions that allowed efficient depletion of endogenous full-length $\mathrm{U} 4$ and concomitant inhibition of splicing, we attempted to restore splicing activity by addition of IVT U4. Titration of IVT U4, from 28-2830 nM, showed effective restoration of splicing relative to depleted extract (Fig. 2A, cf. lanes 3-9 to lane 2). Maximum reconstitution was achieved by addition of $283 \mathrm{nM}$ IVT U4 (lane 6). This U4 concentration corresponds to about 300 -fold more than

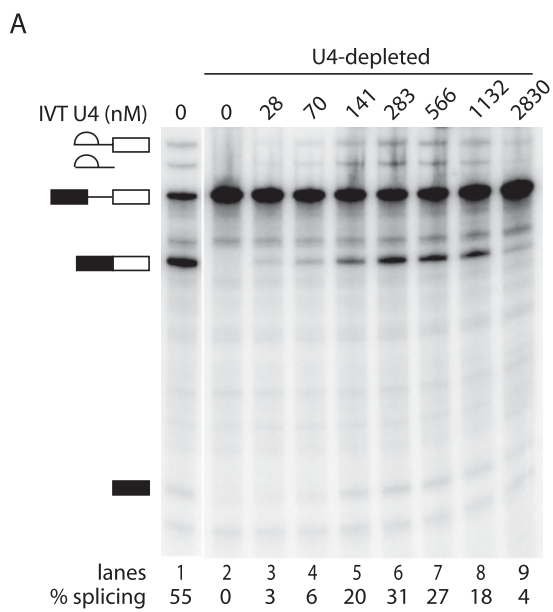

B

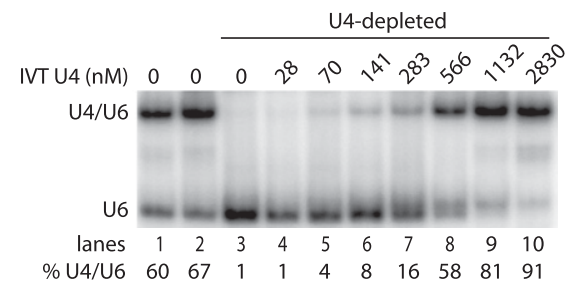

FIGURE 2. Reconstitution of splicing activity in U4-depleted extract by IVT $\mathrm{U} 4$ is not limited by the extent of base-paired U4/U6 formation. (A) Maximum splicing reconstitution is obtained with addition of $283 \mathrm{nM}$ IVT U4. Denaturing splicing gel as in Figure 1D. (Lane 1) Mock-depleted extract. (Lanes 2-9) Depleted extract with IVT U4 added as indicated above lanes. Absolute splicing efficiency of each reaction is indicated below gel. (B) Extent of base-pairing with U6 snRNA increases with added IVT U4. Non-denaturing solution hybridization gel probed using a radioactively labeled oligonucleotide complementary to U6, showing the base-pairing status of U6. (Lane 1) Untreated splicing extract. (Lane 2) Mock-depleted extract. (Lanes 3-10) U4-depleted splicing extract with IVT U4 added as indicated. The percentage of U6 in base-paired complex is shown below the gel. 
endogenous U4 levels (see Discussion) (Brenner and Guthrie 2006). Addition of $>283 \mathrm{nM}$ IVT U4 resulted in decreased splicing; therefore, subsequent experiments used $283 \mathrm{nM} \mathrm{U} 4$.

A range of degradation oligonucleotide concentrations, from $0.05-50 \mu \mathrm{M}$, was examined to identify the optimal concentration for depletion of full-length U4 while maintaining the ability of the depleted extract to restore splicing upon addition of IVT U4. This analysis showed that maximum reconstitution was achieved after depletion with $0.8 \mu \mathrm{M}$ oligonucleotide (data not shown).

To ascertain whether U4/U6 disnRNP levels were limiting for splicing reconstitution, we measured the corresponding U4/U6 levels for each of the splicing reactions in Figure 2A (Fig. 2B). In contrast to the splicing efficiency, which peaked at $283 \mathrm{nM} \mathrm{U} 4, \mathrm{U} 4 / \mathrm{U} 6$ levels continued to increase with increasing U4 to a maximum of $91 \%$ at $2830 \mathrm{nM}$ (lane 10). In untreated splicing extract, $60 \%$ of U6 was base-paired (lane 1) compared with $67 \%$ in mockdepleted extract (lane 2). By comparison, only $16 \%$ of U6 was base-paired when we reconstituted with $283 \mathrm{nM} \mathrm{U} 4$ (lane 7), suggesting that there was insufficient di-snRNP to support maximal splicing. Nevertheless, when 566 nM U4 was added, yielding $58 \%$ basepairing of U6 (lane 8), the splicing efficiency decreased (Fig. 2A, lane 7). Further increases in U4 concentration resulted in further decreased splicing efficiency.
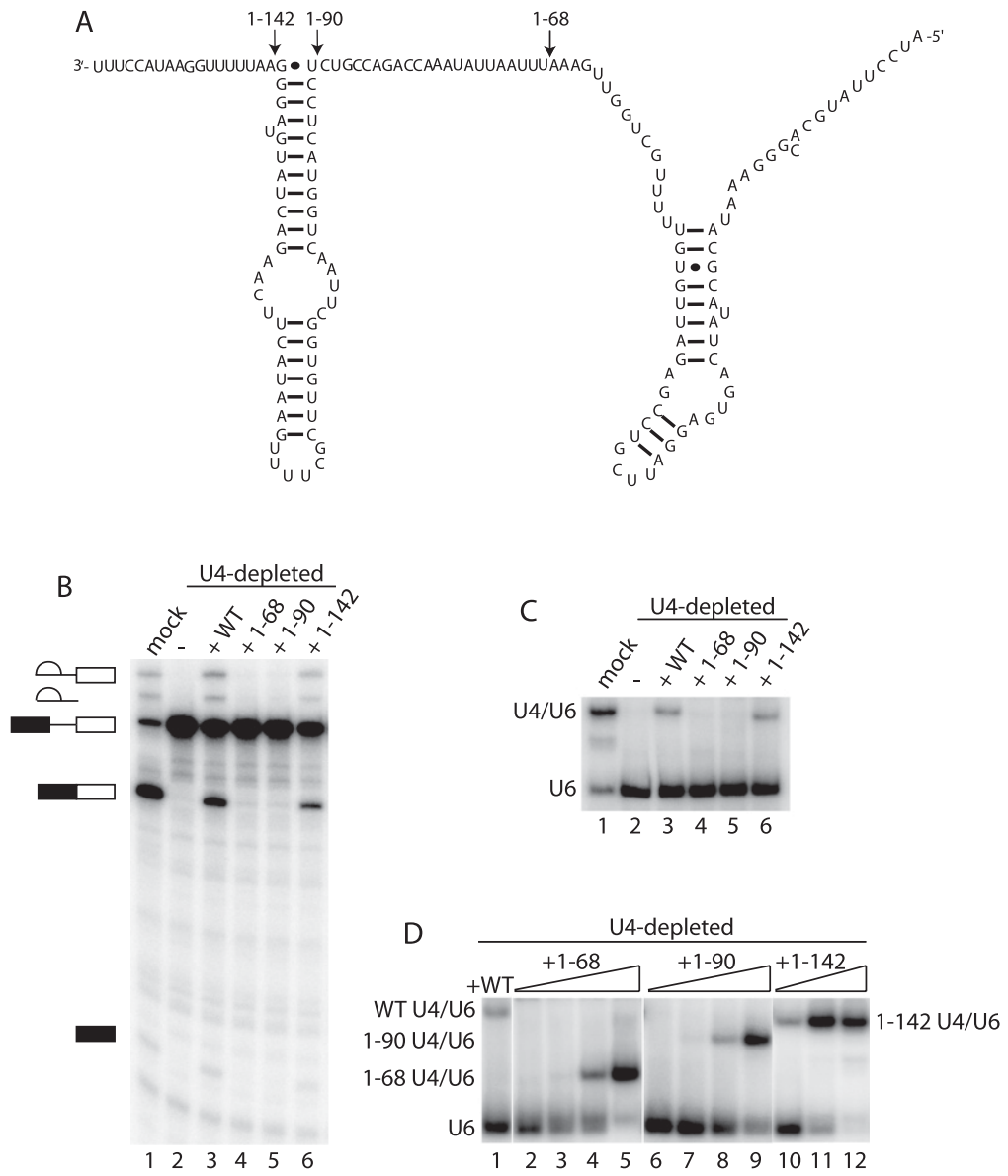

FIGURE 3. The $3^{\prime}$ stem-loop of U4 is required for efficient base-pairing to U6. (A) Secondary structure of U4 snRNA with the $3^{\prime}$ ends of the truncation mutants indicated. (B) U4 truncations lacking the $3^{\prime}$ stem-loop do not support reconstitution of splicing. Denaturing gel analysis of splicing in mock-depleted (lane 1) or U4-depleted (lanes 2-6) extract reconstituted using wild-type (WT) or mutant IVT U4. Splicing substrate, intermediates, and products are indicated to the left of the gel, as in Figure 1. (C) The 3' stem-loop of U4 is required for basepairing to U6 at $283 \mathrm{nM} \mathrm{U} 4$. Non-denaturing solution hybridization gel analysis of the basepairing status of U6 in mock-depleted (lane 1) or U4-depleted (lanes 2-6) extract reconstituted using WT or mutant IVT U4. (D) U4 truncations can be driven into complexes with U6 by increasing their concentration above $283 \mathrm{nM}$. Non-denaturing solution hybridization analysis of extent of U4/U6 formation as in panel C. (Lane 1) $283 \mathrm{nM}$ full-length IVT U4. Increasing concentrations of truncated U4 mutants: 283, 566, 1132, and $2830 \mathrm{nM}$ (1-142 only to $1132 \mathrm{nM})$.

\section{Splicing activities of $U 43^{\prime}$ truncation mutants}

To examine the functional domains of yeast U4, we first determined the minimum sequence that supports U4/U6 formation and splicing using our reconstitution assay with U4 mutants. Given that stems I and II and the 5' stem-loop are all required for efficient spliceosome assembly and splicing in human extract and Xenopus oocytes (Vankan et al. 1990; Wersig and Bindereif 1990, 1992), we tested a mutant containing these three structural features, which consisted of nt 1-68. This mutant, U4 1-68, lacks the Sm binding site, the $3^{\prime}$ stem-loop, and most of the central domain (Fig. 3A); however, it does contain the $5^{\prime}$ portion of the central domain identified by Wersig and Bindereif (1992) and Vankan et al. (1992) as being important for splicing activity.
To examine the effect of truncating U4 at nt 68, U4 1-68 transcript was added to extract depleted of endogenous U4, and its ability to restore splicing was assessed. Unexpectedly, the addition of U4 1-68 to the U4-depleted extract did not result in reconstitution of splicing activity (Fig. 3B, lane 4), in contrast to the ability of wild-type IVT U4 to restore splicing to $21 \%$ from $1 \%$ in the U4-depleted extract (Fig. 3B, lanes 2 and 3; splicing and U4/U6 formation efficiencies reported in the text are averages from at least three measurements; see Table 1).

Lack of splicing reconstitution by U4 1-68 may be due to the absence of the $3^{\prime}$ portion of the central domain (nt 6990). While not essential for splicing, deletion of this sequence from mammalian U4 has an appreciable effect on splicing 
TABLE 1. Di-snRNP formation and splicing reconstitution efficiencies of the $U 4$ mutants

\begin{tabular}{|c|c|c|c|c|c|c|c|}
\hline Mutant & $\begin{array}{l}\text { Di-snRNP } \\
\text { formation }^{\mathrm{a}}\end{array}$ & Splicing ${ }^{\mathrm{b}}$ & Splicing ${ }^{c}$ & $\begin{array}{l}\mathrm{K}_{\mathrm{D}} \\
(\mathrm{nM})\end{array}$ & Error & $\mathrm{R}^{2}$ & $\begin{array}{c}\text { Stability }{ }^{d} \\
(\%)\end{array}$ \\
\hline SE $30^{\prime}$ & & & $81 \pm 8$ & & & & \\
\hline Mock & & & $54 \pm 9$ & & & & \\
\hline DSE & & & $0.6 \pm 0.6$ & & & & \\
\hline Wild type & 100 & 100 & $21 \pm 8$ & 800 & 47 & 0.88 & $45 \pm 18$ \\
\hline $1-68$ & $7 \pm 12$ & 0 & 0 & 1518 & 264 & 0.59 & $26 \pm 14$ \\
\hline $1-90$ & $0 \pm 1$ & 0 & 0 & 1472 & 131 & 0.78 & $31 \pm 9$ \\
\hline $1-142$ & $109 \pm 27$ & $49 \pm 10$ & $8 \pm 2$ & 670 & 34 & 0.90 & $29 \pm 9$ \\
\hline$\Delta 3^{\prime} \mathrm{SL}$ & $3 \pm 6$ & 0 & 0 & 2192 & 175 & 0.82 & $68 \pm 21$ \\
\hline$\Delta \mathrm{USL}$ & $35 \pm 17$ & $6 \pm 2$ & $1 \pm 1$ & 1137 & 75 & 0.92 & $72 \pm 9$ \\
\hline$\Delta 138$ & $105 \pm 57$ & $10 \pm 2$ & $2 \pm 1$ & 650 & 68 & 0.69 & $44 \pm 7$ \\
\hline$\Delta 131-133$ & $60 \pm 33$ & $28 \pm 7$ & $5 \pm 1$ & 836 & 59 & 0.89 & $32 \pm 12$ \\
\hline$\Delta$ bulge & $60 \pm 19$ & $11 \pm 2$ & $2 \pm 1$ & 762 & 33 & 0.95 & $73 \pm 13$ \\
\hline$\Delta 102-130$ & $24 \pm 22$ & $4 \pm 1$ & $1 \pm 1$ & 1105 & 113 & 0.85 & $85 \pm 3$ \\
\hline$\Delta 107-126$ & $8 \pm 9$ & 0 & 0 & 1666 & 157 & 0.86 & $68 \pm 2$ \\
\hline$\Delta 66-90$ & $227 \pm 108$ & 0 & 0 & 390 & 34 & 0.88 & $47 \pm 16$ \\
\hline$\Delta 69-90$ & $168 \pm 48$ & $6 \pm 3$ & $1 \pm 0$ & 470 & 53 & 0.70 & $51 \pm 10$ \\
\hline$\Delta$ loop(AAAAAA) & $12 \pm 9$ & 0 & 0 & 1287 & 107 & 0.94 & $62 \pm 21$ \\
\hline$\Delta$ loop(GGCUU) & $15 \pm 4$ & 0 & 0 & 1851 & 23 & 0.999 & $49 \pm 23$ \\
\hline$\Delta$ loop(UUUUC) & $11 \pm 2$ & 0 & 0 & 1317 & 123 & 0.93 & $57 \pm 22$ \\
\hline
\end{tabular}

${ }^{a} \mathrm{Di}$-snRNP formation values are based on the concentration of $U 4$ that gave the highest reconstitution of splicing using wild-type $U 4,283 \mathrm{nM}$, and are given as a percent of wildtype di-snRNP formation.

${ }^{b}$ Splicing efficiencies are presented as a percentage of the wild type, measured separately in each experiment, and are the average of at least three measurements \pm SD.

${ }^{\mathrm{c}}$ Absolute splicing efficiencies. Values shown are the average over all experiments $(n \geq 3)$.

${ }^{d}$ Stability was measured as a percentage of transcript remaining after incubation in depleted extract under splicing conditions.

to function in splicing is not due to a lack of stability; rather, these mutants lack a functionally essential component of U4.

\section{Interaction of the $\mathrm{U} 43^{\prime}$ truncation mutants with U6}

Despite the fact that $\mathrm{U} 4$ truncation mutants 1-68 and 1-90 contain the only regions of U4 known to interact with U6, their inability to reconstitute splicing in U4-depleted extract could be caused by reduced or absent base-pairing with U6 snRNA. To investigate this possibility, we measured the base-pairing status of U6. As expected, U4 depletion produced extract in which U6 was found exclusively in the free snRNP (Fig. 3C, lane 2). Both wild-type U4 and U4 1-142 were able to reconstitute formation of the di-snRNP in U4-depleted extract to $\sim 20 \%$ of normal levels (Fig. 3C, lanes 3 and 6); however, in the majority of experiments we found that U4 1-142 yielded more U4/U6 than the wild type (Table 1). U4 1-68 and 1-90 were unable to reconstitute di-snRNP formation at the concentration tested (Fig. 3C, lanes 4 and 5).

efficiency (Wersig and Bindereif 1992). We therefore constructed a longer $3^{\prime}$ truncation mutant containing the entire central domain (U4 1-90) (Fig. 3A) and examined its ability to reconstitute U4-depleted extract. Surprisingly, this mutant was also unable to reconstitute splicing (Fig. 3B, lane 5).

The final U4 $3^{\prime}$ truncation mutant we examined, U4 1142 , contains the $3^{\prime}$ stem-loop in addition to the complete central domain, stems I and II, and the $5^{\prime}$ stem-loop and thus lacks only the $3^{\prime}$ terminal domain of U4, which contains the Sm protein binding site (Fig. 3A). Consistent with reconstitution studies in human nuclear extract (Wersig and Bindereif 1992), we found that the $3^{\prime}$ terminal domain of yeast $\mathrm{U} 4$ was not essential for splicing in vitro (Fig. 3B, lane 6 ), although the average level of splicing restored, determined from multiple measurements, was only $49 \%$ of that restored by wild-type U4 (Table 1).

The lack of splicing reconstitution by U4 1-68 and 1-90 could be due to the removal of a functionally essential region of the molecule or to decreased stability of these mutants in splicing extract. To investigate this second possibility, we examined the stability of wild-type and mutant IVT U4 snRNA after incubation in U4-depleted splicing extract. The three mutants were all less stable than wild-type IVT U4, with U4 1-142, which was active in the reconstitution assay, having an intermediate stability (Table 1). This suggests that the inability of the shorter $3^{\prime}$ truncation mutants
The reduced ability of $\mathrm{U} 4$ truncations to form U4/U6 may be due to a kinetic defect in base pair formation or, alternatively, to reduced stability of the base-paired product. To test the first possibility, we allowed the reconstitution reactions to proceed for varying lengths of time up to $36 \mathrm{~min}$. We found that the fraction of U4/U6 did not change after $12 \mathrm{~min}$, indicating that the reactions had reached equilibrium (data not shown). To test the second possibility, we performed titrations of the truncated U4 mutants, from 141 to $2830 \mathrm{nM}$, and subsequently examined U4/U6 levels. When we fit a binding curve to the titration data to calculate an apparent $\mathrm{K}_{\mathrm{D}}$ (see Materials and Methods), we found a significant difference between the mutants, ranging from $1528 \mathrm{nM}$ for 1-68 to $670 \mathrm{nM}$ for 1-142, compared with $800 \mathrm{nM}$ for the wild type (Table 1). These results are consistent with the truncations affecting di-snRNP stability. Surprisingly, the 1-142 mutant appears to have a more stable interaction with U6 than wild-type U4, suggesting that the Sm ring of U4 may antagonize base-pairing with U6.

The ability of U4 1-68 and 1-90 to base pair to U6 at high concentration suggested that they may be able to reconstitute splicing at these concentrations. We therefore tested splicing reconstitution using these mutants at concentrations from 283-2830 nM, which corresponded to U4/U6 levels from $0 \%-98 \%$ for $1-68$ and $0 \%-72 \%$ for $1-90$ (Fig. 3D, lanes 2-9). Neither mutant was able to reconstitute splicing 
at any concentration tested (data not shown). U4 1-142 was similarly tested in a splicing reconstitution titration assay and, like the wild type, showed maximum splicing at $283 \mathrm{nM}$, despite increasing di-snRNP formation up to the highest concentration tested $(1132 \mathrm{nM}$, yielding 93\% di-snRNP; lane 12).

We conclude that the inability of $\mathrm{U} 4$ 1-68 and 1-90 to reconstitute splicing does not stem exclusively from their reduced ability to interact with $\mathrm{U} 6$, as they do not support splicing even under conditions where high levels of U4/U6 are formed. Intriguingly, these experiments point to the $3^{\prime}$ stem-loop of U4 as an important determinant of U4/U6 di-snRNP formation.

\section{Mutational analysis of the $3^{\prime}$ stem-loop of U4 snRNA}

To directly test whether the $3^{\prime}$ stemloop is required for di-snRNP formation, we constructed a mutant, $\Delta 3^{\prime} \mathrm{SL}$, that was missing the entire $3^{\prime}$ stemloop (nt 91-141) (see Fig. 3A) but still contained the Sm binding site. Consistent with the above experiments, this mutant was unable to participate in disnRNP formation at the normal concentration (Fig. 4B, lane 4) and was also unable to restore splicing at this concentration or any other up to $2830 \mathrm{nM}$ (Fig. 4C, lane 4; data not shown). Indeed, $\Delta 3^{\prime}$ SL had the highest $\mathrm{K}_{\mathrm{D}}{ }^{\text {app }}$ of any U4 mutant tested, $2192 \mathrm{nM}$ (Table 1).

Given the unexpected importance of the 3' SL for disnRNP formation and splicing, we undertook a more detailed mutational analysis to identify functionally important regions within this domain. We constructed $10 \mathrm{U} 4$ mutants containing deletions or base mutations throughout the $3^{\prime}$ stem-loop (Fig. 4A) and analyzed the ability of each to restore splicing and di-snRNP formation in U4depleted extract.

To investigate whether the nucleotides important for disnRNP formation are located in the upper portion of the $3^{\prime}$ stem-loop, we examined the effects of a number of deletions in this region. We constructed a mutant, $\Delta U S L$, in which the entire upper stem and central bulge were deleted, leaving only the distal portion of the stem and loop (Fig. $4 \mathrm{~A})$. While this mutant had a $\mathrm{K}_{\mathrm{D}}{ }^{\text {app }}$ of $1137 \mathrm{nM}$ for disnRNP formation, the splicing activity restored was $<10 \%$ that of the wild type (Fig. 4B and C, lane 5; Table 1), indicating that sequences important for both di-snRNP forma-
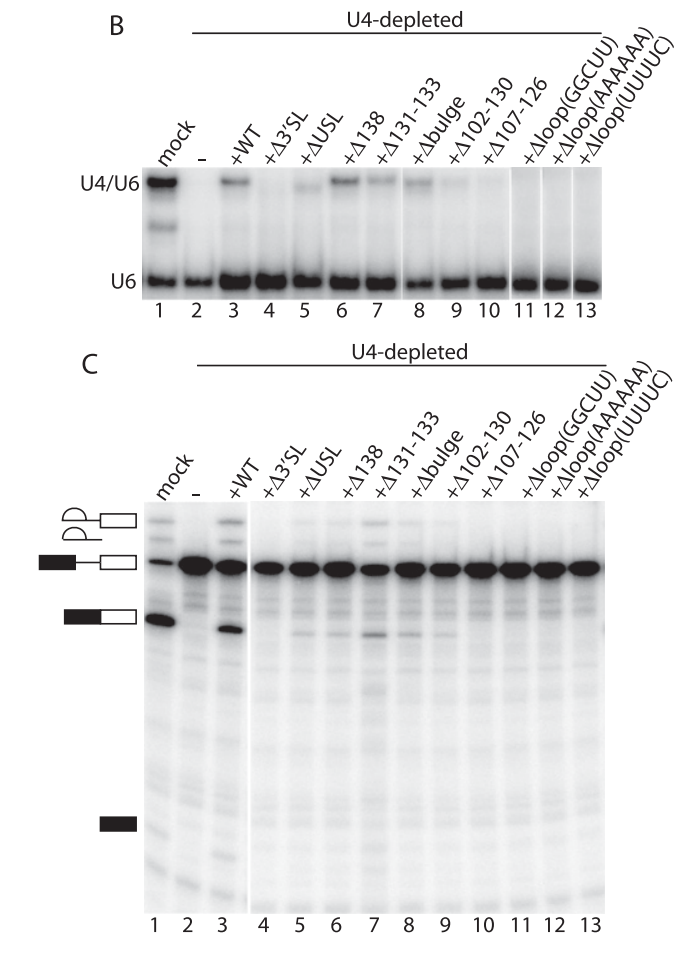

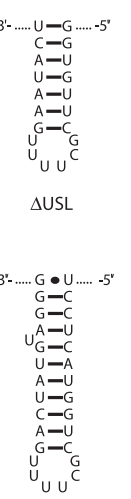

$\Delta 102-130$ (WT loop)

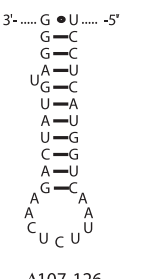

$\Delta 107-126$
FIGURE 4. U4 $3^{\prime}$ stem-loop mutations that form comparable levels of U4/U6 to the wild type (WT) are nevertheless defective in splicing reconstitution. (A) Secondary structures of constructs used in this experiment: WT U4 3' stem-loop, deletion of the 11-nt bulge ( $\Delta$ bulge, the upper stem and bulge ( $\Delta$ USL), the bulge and lower stem $(\Delta 102-130$ [WT loop]), or the lower stem and loop $(\Delta 107-126)$. Locations of deleted $(138 ; 131-133)$ or mutated (loop y positions in U4's 3' stem-loop affect U4/U6 formation. Non-denaturing gel showing the reconstituted using WT 6 in mock-depleted (lane 1) or U4-depleted (lanes 2-13) extract splicing reconstitution. Denaturing gel showing splicing activity in mock-depleted (lane 1) or U4-depleted (lanes 2-13) extract reconstituted using WT or mutant IVT U4 as in B. Splicing substrate, intermediates, and products are indicated to the left of the gel, as in Figure 1. tion and splicing had been deleted. To identify these sequences, we constructed three mutants containing smaller deletions within the upper portion of the $3^{\prime}$ stem-loop. Deletion of U138, a highly conserved bulged nucleotide (Myslinski and Branlant 1991), resulted in better-than-wild-type interaction with U6 $\left(\mathrm{K}_{\mathrm{D}}{ }^{\text {app }}=650 \mathrm{nM}\right)$ but restored only $10 \%$ of the splicing activity seen with wild-type U4 (lane 6; Table 1). In contrast, deletion of nucleotides 131-133, a mutation previously shown to produce a cold-sensitive phenotype in vivo (Hu et al. 1995b), resulted in levels of di-snRNP formation slightly below that of the wild type $(836 \mathrm{nM})$ and splicing levels that were $28 \%$ that of the wild type (lane 7 ; Table 1). Finally, similar to $\Delta 138$, deletion of the 9-nt central bulge of the $3^{\prime}$ stem-loop, $\Delta$ bulge (Fig. 4A), resulted in above-wild-type interaction with U6 (762 nM) but substantially below-wild-type restoration of splicing ( $11 \%$ of the wild type) (lane 8; Table 1).

While deletions within the upper portion of the $3^{\prime}$ stemloop identified nucleotides that contribute to splicing, none of these mutations resulted in the strong block to di-snRNP

RNA, Vol. 18, No. 5 
formation that was produced by deletion of the entire $3^{\prime}$ stem-loop. To investigate whether a sequence essential for di-snRNP formation resides within the lower portion of the 3' stem-loop, we generated five mutants containing deletions within this region. Deletion of the central bulge and distal portion of the stem, leaving the two sides of the upper stem connected by the wild-type loop sequence, produced $\Delta 102-130$ (Fig. 4A). This mutant had an apparent $K_{D}$ for disnRNP formation of $1105 \mathrm{nM}$ and low, but above background, splicing (Fig. 4B,C lane 9; Table 1). Deletion of the entire lower stem and loop, $\Delta 107-126$ (Fig. 4A), severely impaired di-snRNP formation $\left(\mathrm{K}_{\mathrm{D}}{ }^{\text {app }}, 1666 \mathrm{nM}\right)$ and splicing (lane 10; Table 1), indicating that a sequence essential for di-snRNP formation had been deleted.

The observation that the apparent $\mathrm{K}_{\mathrm{D}}$ for U4/U6 formation of $\Delta 102-130$ was substantially lower than $\Delta 107-126$ suggested that the loop nucleotides of the $3^{\prime}$ stem-loop are essential for di-snRNP formation. To test this hypothesis, we generated mutants in which the $6 \mathrm{nt}$ of the loop were replaced. First, we asked whether the sequence of the loop was important by replacing the wild-type $3^{\prime}$-UUUUCG- $5^{\prime}$ sequence with all As. This mutant formed no U4/U6 disnRNP at the standard concentration and, consequently, did not support splicing (Fig. 4B,C, lane 12), indicating that nucleotide identity in the loop is important for U4/U6 formation. An alternative loop sequence from Trypanosoma brucei had previously been shown to form U4/U6 in vivo in the context of a chimeric U4 (Bordonné et al. 1990). We tested this loop sequence, $3^{\prime}$-GGCUU-5' , and found that, as with the all-adenosine loop, it supported neither base pair formation nor splicing (lane 11). Finally, to test whether the length of the loop was important, we made a version lacking G114 at the $5^{\prime}$ end $\left(3^{\prime}\right.$-UUUUC- $\left.5^{\prime}\right)$. Similarly to the other loop mutants, this version of $\mathrm{U} 4$ was inactive in base pair formation and splicing (lane 13). Although all of the 3' stem-loop mutants were able to base pair with U6 at concentrations $>283 \mathrm{nM}$, those that were unable to form disnRNP or splice at $283 \mathrm{nM}$ were also unable to splice at higher concentrations (i.e., $\Delta 3^{\prime}$ SL, $\Delta 107-126$, and all loop mutants; data not shown). Based on these experiments, the loop appears to be the most important portion of the 3' stem-loop for both U4/U6 formation and splicing.

\section{Function of the central domain}

Having demonstrated that the $3^{\prime}$ stem-loop of U4 was required for productive U4/U6 formation (i.e., di-snRNP formation that supports splicing), we sought to determine whether it might also have a function later in the splicing reaction. We attempted to remove the $3^{\prime}$ portion of $\mathrm{U} 4$ after di-snRNP formation using an oligonucleotide targeting the central domain (Fig. 5A). This resulted in separate but stable $5^{\prime}$ and $3^{\prime}$ portions of U4 (data not shown). Degradation was performed under either splicing conditions (in the presence of added pre-mRNA) or nonsplicing condi-
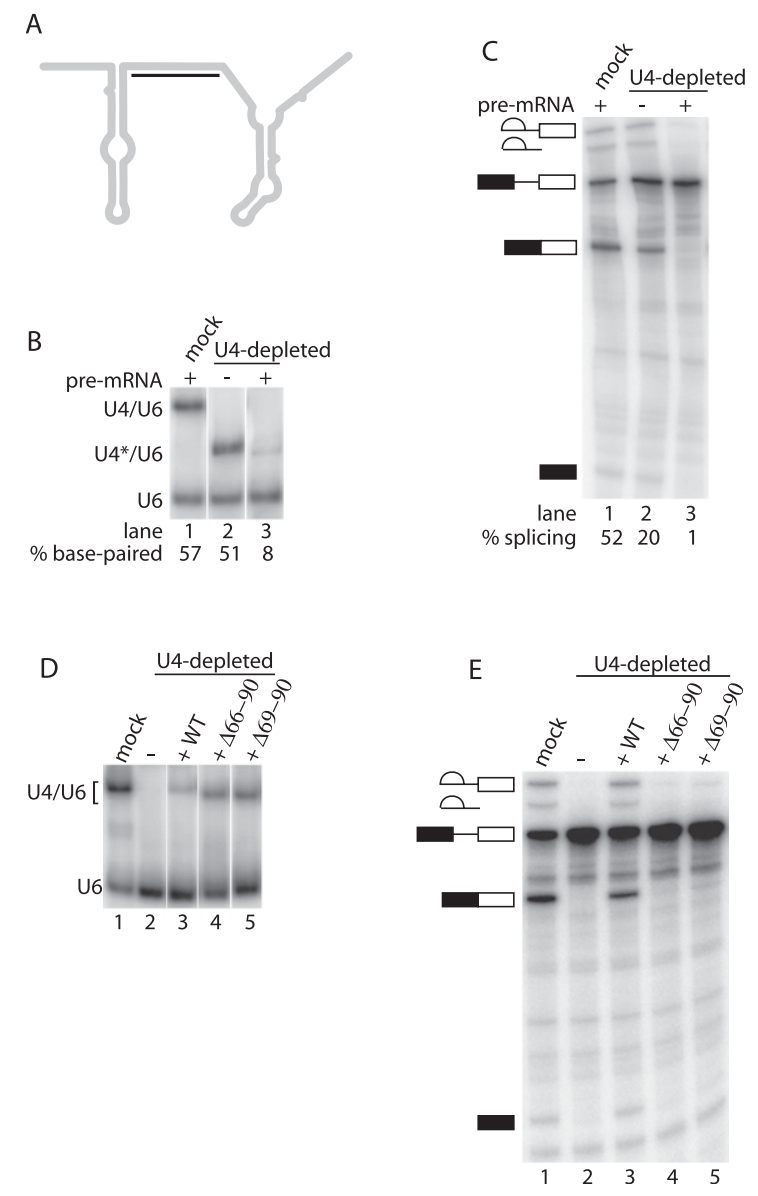

FIGURE 5. The central domain of U4 functions during U4/U6 formation and splicing. (A) Secondary structure of U4 (gray) with targeting oligonucleotide binding site indicated (black line). (B) U4 requires its central domain to re-associate with U6. Non-denaturing solution hybridization gel showing the base-pairing status of U6 in mock-depleted extract (lane 1) or extract depleted of U4 in the absence (lane 2) or presence (lane 3) of added unlabeled actin premRNA. The positions of base-paired wild-type (WT) U4/U6, U6 basepaired to the truncated $5^{\prime}$ portion of $\mathrm{U} 4\left(\mathrm{U} 4^{*} / \mathrm{U} 6\right)$, and free U6 are indicated to the left of the gel. The fraction of U6 base-paired to U4 in each reaction is indicated below the gel. (C) Degraded U4 supports splicing when associated with U6. Denaturing gel showing splicing activity in mock-depleted extract (lane 1) or extract depleted of U4 in the absence (lane 2) or presence (lane 3) of pre-mRNA added during the U4 degradation step. Splicing substrate, intermediates, and products are indicated to the left of the gel, as in Figure 1. Absolute splicing efficiency of each reaction is indicated below the gel. $(D)$ Deletion of U4's central domain increases steady-state levels of U4/U6 di-snRNP. U4/U6 analysis as in $B$ of mock-depleted (lane 1) or depleted (lanes 2-5) extracts reconstituted with no U4 (lane 2) or U4 variants as shown. Note that U4/U6 migrates faster with the mutant $\mathrm{U} 4$ s due to their smaller size. (E) U4's central domain is necessary for splicing. Splicing analysis of the reactions in panel $D$.

tions (in the absence of added pre-mRNA as done previously) (Horowitz and Abelson 1993).

In the absence of targeting oligonucleotide (Fig. 5B, lane 1), the majority of U6 is associated with U4. Similarly, following cleavage of U4 under nonsplicing conditions (lane 2), the majority of $\mathrm{U} 6$ remained associated with the $5^{\prime}$ portion 
of U4 (containing nt 1-64), demonstrating that the complex of U6 with truncated U4 was stable. Cleavage of U4 under splicing conditions, however, resulted in the conversion of almost all U6 to the free snRNA (lane 3). We infer that the truncated U4 and U6 were separated during splicing and were unable to re-anneal. Surprisingly, extract containing the cleaved U4/U6 complex supported subsequent splicing of radioactively labeled pre-mRNA at $\sim 40 \%$ of normal splicing levels (Fig. 5C, lanes 1,2), while extract containing truncated U4 separated from U6 was not able to support splicing (lane 3). These results demonstrate that the $5^{\prime}$ portion of U4 was able to remain in a stable, functional complex with U6 after central domain degradation and that the $3^{\prime}$ stem-loop does not need to be physically attached to the $5^{\prime}$ end of U4 (nt 1-64 in this experiment) after U4/U6 formation. Due to the stability of the $3^{\prime}$ end of $U 4$, these experiments were unable to determine the step at which splicing is blocked in the absence of the $3^{\prime}$ stem-loop.

These results raise the possibility that U4's central domain (Figs. 1A, 3A) is not necessary for $\mathrm{U} 4$ function beyond its role in joining the two functionally essential ends of the molecule. To test this, we reconstituted splicing with two U4 mutants, $\Delta 66-90$ and $\Delta 69-90$, in which the central domain was deleted. Wersig and Bindereif (1992) previously showed that the nucleotides in human U4 corresponding to yeast positions 65-68 contributed to splicing in vitro, so we made one deletion that removed these nucleotides along with the rest of the central domain ( $\Delta 66-90)$, and another $(\Delta 69-90)$ that retained them. Surprisingly, both mutants base-paired with U6 far more than wild-type U4 (Fig. 5D, lanes 3-5). Titrations yielded $\mathrm{K}_{\mathrm{D}}{ }^{\text {app }}$ values of 390 and $470 \mathrm{nM}$, respectively, compared with $800 \mathrm{nM}$ for the wild type (Table 1), the most stable interactions with $\mathrm{U} 6$ of any $\mathrm{U} 4$ mutants that we have tested. When the ability of these mutants to support splicing was measured, however, $\Delta 66-90$ had no activity and $\Delta 69-90$ had very little activity, albeit consistently above depleted extract (Fig. 5E; Table 1), consistent with the observations of Wersig and Bindereif (1992). In contrast to the oligo degradation experiments (Fig. 5B,C), these deletion experiments suggest that the central domain contributes to splicing but antagonizes U4/U6 formation (see Discussion).

\section{snRNP analysis of U4 tri-snRNP formation}

Certain U4 mutants described above were notable for having a lower apparent $\mathrm{K}_{\mathrm{D}}$ than wild-type U4 (U4 1-142, $\Delta 138$, $\Delta$ bulge, $\Delta 66-90$, and $\Delta 69-90)$, yet none of them supported splicing as well as the wild type. One possibility is that these $\mathrm{U} 4$ variants were defective in the next assembly step after U4/U6 di-snRNP formation, namely, U4/U6.U5 tri-snRNP formation. To test this, we measured the ability of these mutants to assemble into tri-snRNPs at several concentrations (Fig. 6).

SnRNP levels in extract can be analyzed on a nondenaturing gel without phenol extracting the samples

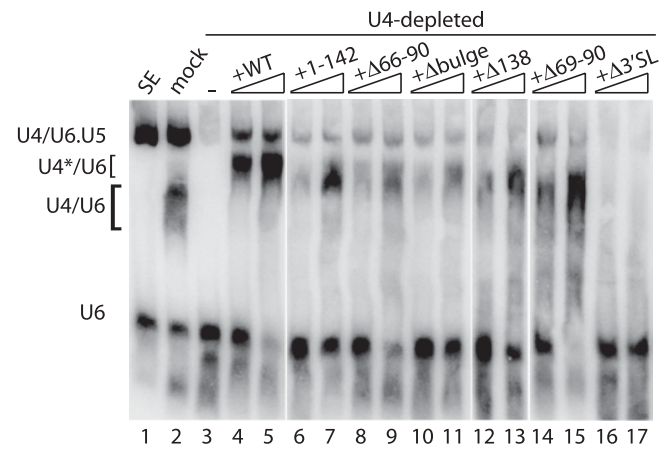

FIGURE 6. U4 deletion mutants are defective in triple-snRNP formation. Non-denaturing Northern blot analysis of unextracted (i.e., protein-containing) samples. Untreated extract (lane 1), mockdepleted extract (lane 2), and depleted extract reconstituted with nothing (lane 3) or with IVT U4 variants (283 or $566 \mathrm{nM}$, lanes 4 17). Location of snRNP species indicated by labels at left. U $4^{\star} / \mathrm{U} 6$ indicates aberrantly migrating U4/U6 di-snRNP seen in all reconstituted extracts.

(i.e., with proteins present) (Cheng and Abelson 1987; Raghunathan and Guthrie 1998a). Under these conditions, all of the base-paired U6 is actually in tri-snRNP, and there is no U4/U6 di-snRNP present (Fig. 6, lane 1; Raghunathan and Guthrie 1998a). Upon addition of ATP, tri-snRNP dissociates into free U4 and U6 snRNPs, which subsequently reform U4/U6 and tri-snRNP (lane 2). Depletion of U4 from extract leads to the complete absence of U4/U6 and a strongly reduced signal at the position where tri-snRNP runs (lane 3). The residual signal at the tri-snRNP position may reflect incomplete degradation of $\mathrm{U} 4$ or may simply be nonspecific background; but in any case, there is no detectable splicing under these conditions (see Fig. 1D).

All of the U4 mutants tested yielded less tri-snRNP after reconstitution than did wild-type $\mathrm{U} 4$, which reconstituted $24 \%$ of the level of tri-snRNP seen in mock-depleted extract. In particular, the five U4 mutants that had lower apparent $\mathrm{K}_{\mathrm{D}} \mathrm{s}$ than the wild type, namely, 1-142, $\Delta 66-90$, $\Delta$ bulge, $\Delta 138$, and $\Delta 69-90$ (lanes $6-15$ ), had $16 \%-27 \%$ as much tri-snRNP as the wild type yet more than $\Delta 3^{\prime} \mathrm{SL}$, which was comparable to the depleted lane (cf. lane 3 to lanes 16,17). The amount of tri-snRNP formed did not increase with additional $\mathrm{U} 4$ above $283 \mathrm{nM}$, in contrast to di-snRNP formation (e.g., cf. lanes 4 and 5), which is consistent with the maximum splicing activity occurring at 283 $\mathrm{nM}$, and may suggest that splicing is limited by tri-snRNP availability.

This demonstrates that all of these mutants are defective in tri-snRNP formation. Notably, they support a wide range of splicing efficiencies, from $49 \%$ of the wild type for U4 $1-142 \%$ to $0 \%$ for $\Delta 66-90$. The lack of correlation between tri-snRNP levels and splicing activity indicates that there is yet another step after tri-snRNP formation in which the $3^{\prime}$ stem-loop and central domain of U4 play a role (see Discussion). 
An important observation is that the di-snRNP present in the reconstituted lanes of the gel in Figure 6 (lanes 4-17) has a lower mobility than di-snRNP from mock-depleted extract (lane 2). We have confirmed that this band, labeled $\mathrm{U} 4{ }^{\star} / \mathrm{U} 6$ in the figure, does not contain U5 snRNA (data not shown), so the aberrant mobility must be either due to a difference in the protein complement compared to untreated extract or due to a conformational change in one or more components (most likely U4). This may in turn be responsible for the reduced levels of tri-snRNP and splicing activity upon reconstitution.

\section{Oligonucleotide accessibility of the $3^{\prime}$ stem-loop of U4 snRNA}

Mutation of specific regions of the $3^{\prime}$ stem-loop of U4 may inhibit di-snRNP formation or subsequent splicing by destabilizing an interaction with a protein. To investigate whether the $3^{\prime}$ stem-loop participates in protein/RNA interactions, we examined the ability of oligonucleotides complementary to the $5^{\prime}$ or $3^{\prime}$ side of this domain (Fig. 7A) to direct degradation of $\mathrm{U} 4$ in extract by RNase $\mathrm{H}$ in the presence or absence of proteins. Surprisingly, the two sides of this domain exhibited unequal oligonucleotide accessibilities. Only the 5 ' side was efficiently degraded in the presence of proteins (Fig. 7B, lanes 2,3), and neither side was susceptible to degradation in the absence of proteins (Fig. 7B, lanes 6,7). Because the Sm site of U4 is known to bind the Sm proteins, this region was used as a degradation control. As expected, it was protected from degradation in the presence of proteins but was accessible to the oligonucleotide, and therefore susceptible to degradation, in the absence proteins (Fig. 7B, lanes 4,8). These results suggest that the $3^{\prime}$ stem-loop of $U 4$ interacts with at least one protein.

A

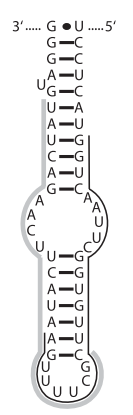

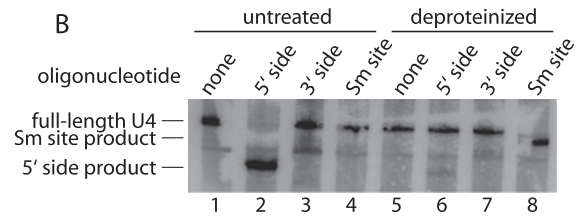

FIGURE 7. Proteins confer asymmetric accessibility to U4's 3' stemloop in untreated splicing extract. $(A)$ Secondary structure of the U4 $3^{\prime}$ stem-loop showing the binding sites of oligonucleotides targeting the $5^{\prime}$ and $3^{\prime}$ sides (black and gray lines, respectively). (B) The U4 3' stem-loop is only degraded in the presence of proteins. Denaturing Northern blot probed for U4, showing U4 size in the absence (lanes 1,5 ) or presence (lanes $2-4,6-8$ ) of oligonucleotide targeting the $5^{\prime}$ or $3^{\prime}$ side of the $3^{\prime}$ stem-loop, or the Sm site. Lanes 1-4 are in the presence of proteins, while lanes 5-8 are in the absence of proteins.

\section{DISCUSSION}

\section{Conditions for successful $\mathrm{U} 4$ reconstitution}

In this study, we have developed the first in vitro splicing reconstitution system for $\mathrm{U} 4 \mathrm{snRNP}$ in yeast. A key requirement of this assay was to deplete endogenous U4 snRNA under conditions of active splicing so that the $5^{\prime}$ end of U4 targeted for RNase $\mathrm{H}$ degradation was no longer base-paired to U6 and was therefore accessible to the targeting DNA oligonucleotide. We were able to reconstitute the splicing activity of the depleted extract by addition of IVT U4, which allowed us to discover an unexpected role for the $3^{\prime}$ stem-loop in di-snRNP formation, as well as in later steps of spliceosome assembly (steps at which U4 3' domains may function are summarized in Fig. 8).

The amount of splicing reconstitution we observed was somewhat lower than that seen with other snRNAs (our range was $21 \%-76 \%$ of the mock reaction, compared with $50 \%-100 \%$ of untreated extract for U6 [Fabrizio et al. 1989], and $55 \%-70 \%$ of the mock reaction for U2 [McPheeters et al. 1989]), and required higher concentrations of IVT U4 (283 nM compared to 40-125 nM for U6 and U2 [Fabrizio et al. 1989; McPheeters et al. 1989; McGrail et al. 2006] and $125 \mathrm{nM}$ for U5 [McGrail and O’Keefe 2008]). Approximately $50 \%$ of the added IVT U4 was degraded in extract over the course of splicing reconstitution. In addition, the aberrant mobility of the reconstituted di-snRNP on a non-denaturing gel (Fig. 6) suggests that much of the IVT U4 is either misfolded or does not associate properly with proteins. Nevertheless, its ability to reconstitute tri-snRNP with normal mobility, as well as splicing activity, suggests that some fraction of the U4 interacts normally with the rest of the splicing machinery.

The decrease in reconstitution efficiency seen with IVT U4 concentrations $>283 \mathrm{nM}$ may be due to increased competition for U4-specific proteins, effectively diluting the proteins available such that fewer U4 RNA molecules receive the full protein complement required for functionality. Alternatively, an inhibitory component in the IVT solution (such as residual acrylamide) could be responsible for the inhibition.

Conditions for complete depletion of endogenous U4 from extracts had to be determined for each extract tested, requiring 15-80 fmol of actin, compared with 4 fmol for a standard splicing reaction. This may reflect the need to cycle the entire pool of endogenous U4 through the splicing pathway, perhaps multiple times, to ensure complete degradation. We cannot explain the variability between extracts. It was also surprising that U4 depletion was only successful under conditions of active splicing, as ATP alone is known to cause the dissociation of tri-snRNP in yeast as well as human extract (Berget and Robberson 1986; Raghunathan and Guthrie 1998b). This suggests the possibility that U4 released from the spliceosome is in a different conformation 
Hayduk et al.

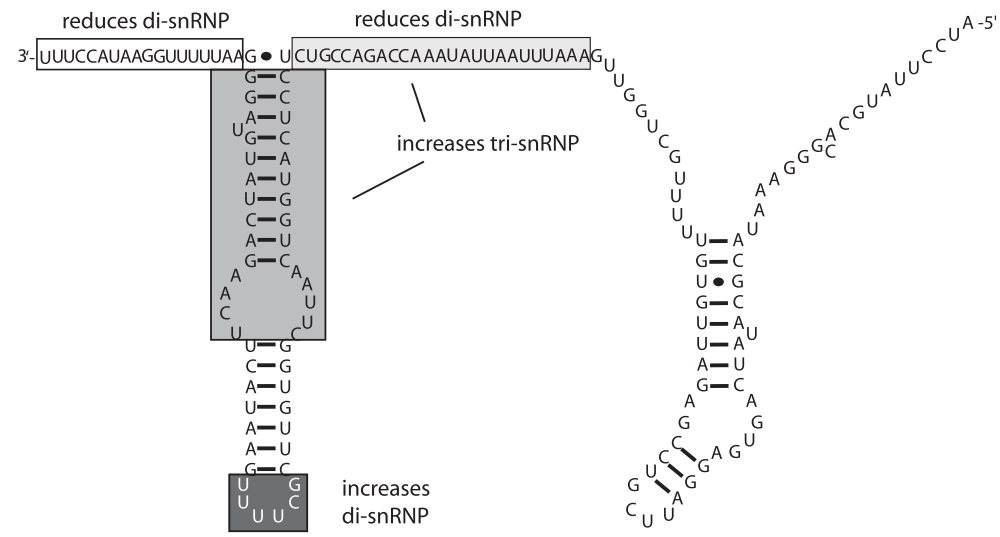

does not significantly inhibit di-snRNP formation (Vankan et al. 1990; 1992; Wersig and Bindereif 1990; 1992). Indeed, these studies further demonstrated this domain to be dispensable for spliceosome assembly and even for splicing, although efficiency was reduced compared with wild-type U4. Similarly, a series of point mutations throughout the 3 ' stem-loop of yeast U4 were found to be functional in vivo, although deletion of nt 131-133 did result in a coldsensitive phenotype ( $\mathrm{Hu}$ et al. 1995b).

In contrast to these studies suggesting that the $3^{\prime}$ stem-loop is not a functionally important element of U4 snRNA, an in vivo analysis by Bordonné et al. (1990) found that substitution of the yeast U4 3' stem-loop with the comparable region of $T$. brucei $\mathrm{U} 4$, an 11-nt stem-loop, decreased interaction with U6 by $60 \%-70 \%$ and was lethal. Similarly, the $3^{\prime}$ stem-loop of human U4atac snRNA has been shown to be required for in vivo activity (Shukla et al. 2002). Hence, the requirement for the U4 $3^{\prime}$ stem-loop appears to vary between organisms and even between the major and minor spliceosome. Furthermore, while the $3^{\prime}$ stem-loop of yeast U4 is clearly necessary for di-snRNP formation, there appears to be considerable flexibility in the sequence requirements of this domain.

The U4 3' stem-loop mutants can be divided into two categories based on their di-snRNP formation and splicing reconstitution efficiencies: those that restored reduced levels of di-snRNP and splicing, and those that restored in-

from U4 released from tri-snRNP under nonsplicing conditions, with the $5^{\prime}$ end of the former being more accessible than the latter. Another possibility is that the difference in oligo accessibility is due to differences in protein composition of the two types of free U4 snRNP.

\section{The $3^{\prime}$ stem-loop of yeast $U 4$ is required for efficient di-snRNP formation}

Reconstitution by U4 $3^{\prime}$ truncation mutants showed that the $3^{\prime}$ stem-loop plays a critical role in promoting the interaction with U6 that is required for subsequent reconstitution of splicing. This was unexpected, as a number of previous mutational analyses of $\mathrm{U} 4$ in human nuclear extract and Xenopus oocytes have shown that deletion of this domain creased levels of di-snRNP but reduced levels of splicing. We note that the two classes of $3^{\prime}$ stem-loop mutants appear to segregate by location in the stem-loop: Those that increase the apparent $K_{D}$ are in the lower stem-loop, while those that have no significant effect $(\Delta 131-133)$ or reduce the apparent $K_{D}(\Delta 138$ and $\Delta$ bulge $)$ are in the upper stem.

Two lines of evidence demonstrate the importance of the loop nucleotides of U4's 3' stem-loop (nt 114-119) in disnRNP formation. First, deletion of either the upper stem $(\Delta \mathrm{USL})$ or the lower stem $(\Delta 102-130)$ has only a modest effect on the apparent $\mathrm{K}_{\mathrm{D}}$ for U4/U6 formation. Importantly, neither of these changes the loop sequence. In contrast, $\Delta 107-126$, which removes the loop along with the lower stem, dramatically increases the apparent $K_{D}$. Second, mutations in the loop itself have a greater impact 
on U4/U6 formation than deletion of either the upper or lower stem, and one of the loop mutants has the secondhighest apparent $\mathrm{K}_{\mathrm{D}}$ of all of the mutations tested, exceeded only by deletion of the entire $3^{\prime}$ stem-loop. Given that di-snRNP must form prior to spliceosome assembly, any mutations that strongly reduce di-snRNP formation will also block splicing. Consistent with this, the only mutations tested that eliminated splicing were those that changed the $3^{\prime}$ loop, with the exception of $\Delta 66-90$, which removes central domain nucleotides previously shown to be important for splicing (Wersig and Bindereif 1992).

The loop mutations we have tested indicate that both the length and the sequence of the loop are important. Likewise, the chimeric U4 with an altered loop sequence discussed above had a strong effect on di-snRNP formation in vivo (Bordonné et al. 1990). The even more dramatic reduction in U4/U6 levels seen when we placed this loop sequence ( $\Delta$ loop(GGCUU)) on the U4 wild-type stem may reflect differences between the in vitro and in vivo assays used or may be a consequence of differences in the stem. In contrast, one of the U4 point mutations analyzed in vivo by $\mathrm{Hu}$ et al. (1995b), G114U, falls within the loop of the 3' stem-loop. Alone, this mutation did not produce a phenotype, suggesting either that our deletion of this nucleotide ( $\Delta \operatorname{loop}($ UUUUC) ) impacts function more than mutations at this position or that the in vitro assay is more sensitive.

\section{The $3^{\prime}$ stem-loop functions at a late stage of spliceosome assembly}

Previous studies of U4 function have mostly focused on its role in di-snRNP formation; however, there is evidence indicating that it plays a role in subsequent steps, such as trisnRNP formation. In yeast, humans, and Xenopus, deletion of the $5^{\prime}$ stem-loop results in wild-type or better di-snRNP formation but invariably inhibits tri-snRNP formation and splicing (Bordonné et al. 1990; Wersig and Bindereif 1992; Wersig et al. 1992). We also tested the effect of deletion of U4's 5' stem-loop in our reconstitution system and similarly found increased levels of U4/U6, but no splicing (data not shown). The $5^{\prime}$ stem-loop of U4 is associated with Prp4, which has been implicated in tri-snRNP formation (Bordonné et al. 1990). In addition, mutations in the second WD repeat of Prp4 block dissociation of U4 from the spliceosome, suggesting yet another role for U4 just prior to spliceosome activation (Ayadi et al. 1997).

An unexpected late function (i.e., after di-snRNP formation) of U4's 3' stem-loop is suggested by the second class of $3^{\prime}$ stem-loop mutants. These mutations, $\Delta 138$ and $\Delta$ bulge, restored similar levels of di-snRNP formation as the wild-type sequence and reduced apparent $K_{D} s$, yet reduced splicing, indicating that they impair a process subsequent to base-pairing with U6. One possible explanation for the observed elevation of di-snRNP levels is that these mutations strengthen interactions with a splicing factor, such as Prp24, that promotes di-snRNP formation. Such gainof-function mutations are, however, relatively rare, and it would be surprising to find even one, let alone two, in the relatively limited survey of mutations described here. Alternatively, these mutations may disrupt an interaction that would normally lead to destabilization of U4/U6, namely, unwinding by the Brr2 helicase prior to spliceosome activation. In this case, we would expect to see an accumulation of spliceosomes or multi-snRNP particles (larger than di-snRNP) (Raghunathan and Guthrie 1998b), yet we do not. The remaining possibility is that these residues may play a role in formation or stabilization of the U4/U6.U5 tri-snRNP. We favor this role for the nucleotides on the $3^{\prime}$ side of the upper stem-loop because our analysis of snRNP particles shows an accumulation of di-snRNP and reduced tri-snRNP. One mechanism by which this region of the $3^{\prime}$ stem-loop could promote tri-snRNP formation could be through interaction with a U5 protein.

\section{The $3^{\prime}$ side of the $3^{\prime}$ stem-loop may bind a protein}

Our oligonucleotide accessibility data showed strong protein dependence. For an RNA sequence to be degraded by RNase $\mathrm{H}$, it must be available for base-pairing with a complementary DNA oligonucleotide. Binding of this targeting oligonucleotide may be blocked if the RNA sequence is involved in secondary or tertiary structure interactions or in protein binding. In the absence of proteins, the $3^{\prime}$ stem-loop of yeast U4 is expected to adopt a highly stable secondary structure with an estimated melting temperature of $77^{\circ} \mathrm{C}$ (Owczarzy et al. 2008). Given that the estimated melting temperatures of the oligonucleotides targeting the $5^{\prime}$ and $3^{\prime}$ sides of the $3^{\prime}$ stem-loop are substantially lower $\left(45^{\circ} \mathrm{C}\right.$ and $43^{\circ} \mathrm{C}$, respectively), it is not surprising that they were not able to direct RNase $\mathrm{H}$ degradation in the absence of proteins.

The differential accessibility of the two sides of the $3^{\prime}$ stem-loop in the presence of proteins was unexpected. The accessibility of the $5^{\prime}$ side demonstrates that the binding of proteins to U4 changes the secondary structure of the $3^{\prime}$ stem-loop. This may result from a protein binding directly to the $3^{\prime}$ side of the stem, disrupting base-pairing within the stem and thereby protecting the $3^{\prime}$ side while releasing the $5^{\prime}$ side for base-pairing with a targeting oligonucleotide. Notably, a recent crystal structure of the U4 core (the Sm ring with U4's Sm binding site and flanking stemloops) supports the possibility of a protein binding the protected side of the $3^{\prime}$ stem-loop (Leung et al. 2011). For example, the structure shows that the conserved bulged $U$ in the $3^{\prime}$ stem-loop (U114 in humans, U138 in S. cerevisiae) is positioned away from the Sm ring such that it would be accessible to a protein. The asymmetric pattern of oligonucleotide accessibility is also consistent with the asymmetry of 
mutational effects observed in previous studies ( $\mathrm{Hu}$ et al. 1995b).

\section{Role of U4's central domain}

As discussed above, our data indicate an unexpected role for the $3^{\prime}$ stem-loop after di-snRNP formation. Surprisingly, the $3^{\prime}$ stem-loop does not have to be connected to the $5^{\prime}$ portion of U4 to carry out this role. Extract in which the central domain of U4 has been completely degraded, leaving stable $5^{\prime}$ and $3^{\prime}$ portions of the snRNA, is still able to support splicing (Fig. 5C; Horowitz and Abelson 1993), implying that once the di-snRNP has formed, separation of the two halves of U4 does not prevent any additional interactions that may occur between them. Surprisingly, deletion of the central domain (nt 66-90 or 69-90) results in higher steady-state levels of U4/U6, suggesting that the central domain antagonizes U4/U6 formation. The higher levels could be due to faster di-snRNP formation, slower tri-snRNP formation, or slower unwinding during spliceosome activation (Fig. 8). Given the accumulation of U4/U6 on snRNP gels and the relatively low levels of tri-snRNP after reconstitution with central domain deletion mutants, we favor the interpretation that the most important function of the central domain occurs during addition of $\mathrm{U} 5$ to the U4/U6 di-snRNP. Taken together, these experiments demonstrate that during U4/U6 formation, the central domain is only required to keep the $3^{\prime}$ stem-loop physically attached to U4, while during tri-snRNP formation, the central domain is required to maintain appropriate spacing between the $5^{\prime}$ region and the $3^{\prime}$ stem-loop of U4.

Our results are consistent with previous work on the central domain. In Xenopus, deletion of the central domain had no effect on U4/U6 formation but blocked splicing (Vankan et al. 1990). Genetic experiments in yeast also suggested that the requirements for the central domain are flexible: Deletion of nt 62-88 had only a mild cold-sensitive phenotype (Hu et al. 1995b), and insertion of an extra $76 \mathrm{nt}$ into the central domain had no effect on growth $(\mathrm{Hu}$ et al. 1995a).

More detailed analysis of the central domain has shown that only the $5^{\prime}$ end has a strong sequence requirement. In Xenopus, substitution of the first 3 nt (64-66) blocked splicing, but no other 3-nt substitution in the central domain had a strong effect (Vankan et al. 1992). Similarly, small deletions throughout the central domain of human U4 revealed that only the $5^{\prime}$ end (nt 64-67) was important for efficient splicing, whereas other deletions reduced splicing by at most 50\% (Wersig and Bindereif 1992).

The ability of the two halves of $\mathrm{U} 4$ in the splicing extract to separate, and the inability of the separated halves to reassociate with U6, allows us to estimate the fraction of splicing in a normal assay that occurs in the initial round and the fraction that occurs after recycling of U6 (i.e., dissociation and reassembly) (Raghunathan and Guthrie 1998a,b). The data in Figure 5C demonstrate that separating the $5^{\prime}$ and $3^{\prime}$ portions of U4 reduces splicing by $\sim 60 \%$, suggesting that the remaining $40 \%$ of a standard in vitro splicing yield is catalyzed prior to recycling. Importantly, cleavage of the central domain of U4 thus provides a practical method to study single turnover splicing kinetics.

\section{Role of the $3^{\prime}$ terminal domain}

Our titration analysis of $\mathrm{U} 4$ allowed us to make quantitative comparisons between the mutants. For example, it is notable that deletion of the $3^{\prime}$ stem-loop gave a higher apparent $\mathrm{K}_{\mathrm{D}}$ than truncation of $\mathrm{U} 4$ at nt $68(2192 \mathrm{nM}$ vs. $1518 \mathrm{nM}$ ), which appears to suggest that removing all of $U 4$ after nt 68 has less impact on U4/U6 formation than simply deleting the $3^{\prime}$ stem-loop. The observation that U4 1-90 has a similar apparent $K_{D}$ to U4 1-68 indicates that the lower $K_{D}$ of U4 1-68, compared with that of $\Delta 3^{\prime} \mathrm{SL}$, is not due to the absence of the central domain and must therefore be due to the absence of the $3^{\prime}$ terminal domain (nt 143-160). We conclude that the $3^{\prime}$ terminal domain serves to reduce steady-state levels of $\mathrm{U} 4 / \mathrm{U} 6$, because its presence in $\Delta 3^{\prime} \mathrm{SL}$ increases the apparent $\mathrm{K}_{\mathrm{D}}$ relative to U4 1-90. Our observation that U4 1-142 has a lower apparent $\mathrm{K}_{\mathrm{D}}$ than wild-type U4 is consistent with this conclusion.

The function of the $3^{\prime}$ terminal domain in decreasing $\mathrm{U} 4 / \mathrm{U} 6$ levels could be due to direct antagonism of the rate of di-snRNP formation or to acceleration of U4/U6 unwinding during spliceosome assembly. Our snRNP gel analysis, which shows no change in tri-snRNP levels between any of the mutants, is more consistent with the former possibility, although it is unclear how the $3^{\prime}$ terminal domain, which includes the Sm protein binding site, would slow disnRNP formation.

The data presented here reveal the unexpected role played by the $3^{\prime}$ stem-loop of yeast U4 in promoting U4/ U6 formation and tri-snRNP assembly, with the loop nucleotides promoting di-snRNP formation and with the upper stem and central domain promoting tri-snRNP formation (Fig. 8). The $3^{\prime}$ terminal domain, which includes the Sm protein binding site, appears to slow the rate of disnRNP formation. Interestingly, a recent article (He et al. 2011) reports that a mutation in the analogous stem of human U4atac is linked to microcephalic osteodysplastic primordial dwarfism type I (MOPD I), also known as Taybi-Linder Syndrome (TALS). Our data suggest that the U4atac mutation may cause problems in U4/U6 formation or in subsequent tri-snRNP formation. These results emphasize the need for structural information to reveal the nature of the conformational changes that occur during RNA structural rearrangements, such as in the transition from free $\mathrm{U} 4$ to the U4/U6 di-snRNP. A thorough understanding of this conformational change will require both static structures as well as information about the dynamic processes that occur during this transition. 


\section{MATERIALS AND METHODS}

\section{DNA oligonucleotides}

U4-targeting oligonucleotides, primers used in site-directed mutagenesis, and U4 and U6 probes are listed in Table 2.

\section{Synthesis of U4 snRNA and actin pre-mRNA}

Wild-type U4 was synthesized from StyI-linearized pT7U4 plasmid template (Ghetti et al. 1995). Templates for synthesis of the U4 mutants were generated by site-directed mutagenesis from pT7U4 plasmid. The same forward primer containing the T7 promoter, oligonucleotide $\mathrm{A}$, was used for all mutants, and the reverse primers are listed in Table 2. PCR products were purified by electrophoresis through a denaturing polyacrylamide gel and visualized by ultraviolet shadowing. Following homogenization in a $1.5-\mathrm{mL}$ microcentrifuge tube using a small pestle (Kontes

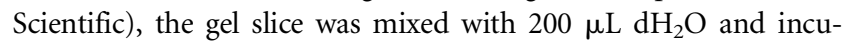
bated for $10 \mathrm{~min}$ at $70^{\circ} \mathrm{C}$. The gel slurry was passed over a DTR column (Edge BioSystems) according to the manufacturer's instructions. DNA was ethanol precipitated and quantified using a NanoDrop spectrophotometer (NanoDrop Technologies). Wildtype U4 and U4 mutants were transcribed in vitro from plasmid or PCR template using the MEGAshortscript Kit (Ambion) according to the manufacturer's instructions. RNA was gel purified and quantified as described for PCR products.

Unlabeled actin pre-mRNA was synthesized from HindIIIlinearized JPS149 plasmid (Vijayraghavan et al. 1986; Mayas et al. 2006) using the MEGAshortscript Kit (Ambion) according to the manufacturer's instructions and was gel purified and quantified as described above. Radioactively labeled actin pre-mRNA was transcribed and purified as previously described (Aukema et al. 2009).

\section{Oligonucleotide-directed RNase $\mathrm{H}$ degradation of $\mathrm{U} 4$}

Whole-cell extract was prepared from protease-deficient yeast strain BJ2168 as described (Ansari and Schwer 1995). U4 degradation reactions were performed in $8 \mu \mathrm{L}$ containing $60 \mathrm{mM} \mathrm{KPO}_{4}(\mathrm{pH} 7.0)$,

TABLE 2. DNA oligonucleotides used in this study

\begin{tabular}{|c|c|c|}
\hline & Sequence $\left(5^{\prime}\right.$ to $\left.3^{\prime}\right)$ & Mutant \\
\hline \multicolumn{3}{|l|}{ PCR primers } \\
\hline A & AATTAATACGACTCACTATAGGGATCCTTATGCACGGGAAATA ${ }^{a}$ & All \\
\hline B & TTTCAАCСAGCAAAAACACA & $1-68$ \\
\hline $\mathrm{C}$ & GACGGTCTGGTTTATAATTAAATTTCA & $1-90$ \\
\hline $\mathrm{D}$ & СССТАCATAGTCTTGAAGTATTCA & $1-142$ \\
\hline $\mathrm{E}$ & AAAGGTATTCCAAAAATTGACGGTCTGGTTTATAATTAAATTTCA & $\Delta 3^{\prime} \mathrm{SL}$ \\
\hline $\mathrm{F}$ & AAAGGTATTCCAAAAATTAGTATTCAAAAGCGAACACCGACGGTCTGGTTTATAATTAAATTTCA & $\Delta \mathrm{USL}$ \\
\hline G & AAAGGTATTCCAAAAATTCCCTCATAGTCTTGAAGTATTC & $\Delta 138$ \\
\hline $\mathrm{H}$ & AAAGGTATTCCAAAAATTCCCTACATATTGAAGTATTCAAAAGCG & $\Delta 131-133$ \\
\hline I & AAAGGTATTCCAAAAATTCCСTACATAGTCAGTATTCAAAAGCGAACACCGACCATGAGGAGACGG & $\Delta$ bulge \\
\hline J & AAAGGTATTCCAAAAATTCCCTACATAGTCAAAAGCGACCATGAGGAGACGG & $\Delta 102-130$ \\
\hline K & AAAGGTATTCCAAAAATTCССТАСАTAGTCTTGAGAATTGACCATGAGGAGAC & $\Delta 107-126$ \\
\hline $\mathrm{L}$ & AAAGGTATTCCAAAAATTCССТACATAGTCTTGAAGTATTCTTTTTTGAACACCGAATTGACCAT & $\Delta$ loop (AAAAAA) \\
\hline M & AAAGGTATTCCAAAAATTCССТACATAGTCTTGAAGTATTCAAGCCGAACACCGAATTGACCAT & $\Delta$ loop (GGCUU) \\
\hline $\mathrm{N}$ & AAAGGTATTCCAAAAATTCCCTACATAGTCTTGAAGTATTCGAAAAGAACACCGAATTGACCAT & $\Delta$ loop (UUUUC) \\
\hline \multirow[t]{3}{*}{$\mathrm{O}$} & 5'-AGATTGTGTTTTTGCTGGTTGTCCTCATGGTCAATTCGG (inside fwd) & $\Delta 66-90$ \\
\hline & 5'-CCGAATTGACCATGAGGACAACCAGCAAAAACACAATCT (inside rev) & \\
\hline & 5'-AAAGGTATTCCAAAAATTCCCTAC (outside rev) & \\
\hline \multirow[t]{3}{*}{$\mathrm{P}$} & 5'-TGTGTTTTTGCTGGTTGAAATCCTCATGGTCAATTCGG (inside fwd) & $\Delta 69-90$ \\
\hline & 5'-CCGAATTGACCATGAGGATTTCAACCAGCAAAAACACA (inside rev) & \\
\hline & 5'-AAAGGTATTCCAAAAATTCCCTAC (outside rev) & \\
\hline \multirow{2}{*}{\multicolumn{3}{|c|}{$\begin{array}{l}\text { U4-targeting } \\
\quad \text { oligonucleotides }\end{array}$}} \\
\hline & & \\
\hline 5'end & CTGATATGCGTATTTCCCGTGCATAAGGAT & \\
\hline Central domain & CGGTCTGGTTTATAATTAAATTTC & \\
\hline $5^{\prime}$ side of $3^{\prime} \mathrm{SL}$ & TCAAAAGCGAACACCGAATTGACC & \\
\hline $3^{\prime}$ side of $3^{\prime} \mathrm{SL}$ & ATAGTCTTGAAGTATTCAAAAGCG & \\
\hline Sm site & AGGTATTCCAAAAATTCCСТAC & \\
\hline \multicolumn{3}{|l|}{ Probes } \\
\hline U1 & CAGTAGGACTTCTTGATC & \\
\hline U2 (L15) & CAGATACTACACTTG & \\
\hline $5^{\prime} \cup 4(\mathrm{CM} 6)$ & TCAACCAGCAAAAACACAATCTCG & \\
\hline $3^{\prime} \cup 4(14 B)$ & AGGTATTCCAAAAATTCCСТAC & \\
\hline U5 (7SmWTNR) & AAGTTCCAAAAAATATGGCAAGC & \\
\hline U6-467 & ATTGTTTCAAATTGACC & \\
\hline U6-6D & AAAACGAAATAAATCTCTTTG & \\
\hline
\end{tabular}

${ }^{\mathrm{a}} \mathrm{T} 7$ promoter indicated in bold. 
$2.5 \mathrm{mM} \mathrm{MgCl} 2,3 \%$ PEG 8000, 50\% (v/v) yeast extract, $10 \mathrm{mM}$ ATP, $0.8 \mu \mathrm{M}$ targeting oligonucleotide ( $5^{\prime}$ end or central domain) (Table 2), and 15-80 fmol unlabeled IVT actin pre-mRNA (depending on the splicing extract). No exogenous RNase $\mathrm{H}$ was added as yeast extract contains sufficient endogenous RNase $\mathrm{H}$ activity. Reactions were incubated for $30 \mathrm{~min}$ at $30^{\circ} \mathrm{C}$. Oligonucleotide accessibility of the $3^{\prime}$ stem-loop and $\mathrm{Sm}$ site was examined as described above except $15 \mu \mathrm{M}$ targeting oligonucleotide (Table 2) was used. For oligonucleotide accessibility of deproteinized U4, yeast extract was phenol/chloroform extracted, ethanol precipitated, and resuspended in $2 \times$ RNase $\mathrm{H}$ buffer $(40 \mathrm{mM}$ Tris- $\mathrm{HCl}$ at $\mathrm{pH} 7.5$, $10 \mathrm{mM} \mathrm{MgCl}_{2}, 4 \mathrm{mM}$ DTT, $0.012 \%$ BSA). Four microliters of this resuspension, containing approximately the amount of $\mathrm{U} 4$ found in $4 \mu \mathrm{L}$ extract, was mixed with targeting oligonucleotide, $2 \mathrm{U}$ RNase $\mathrm{H}$ (Ambion), and $10 \mathrm{mM}$ ATP to a volume of $8 \mu \mathrm{L}$ and incubated as described above.

\section{Northern blot analysis of $\mathrm{U} 4$ degradation and IVT U4 stability}

U4 degradation reactions were terminated by addition of $200 \mu \mathrm{L}$ stop buffer (0.3 M sodium acetate, $1 \mathrm{mM}$ EDTA, $0.1 \%$ SDS, $34 \mu \mathrm{g} / \mathrm{mL}$ Escherichia coli tRNA). Reactions were phenol/chloroform extracted, ethanol precipitated, and electrophoresed through a $6 \%$ (19:1) $7 \mathrm{M}$ urea denaturing polyacrylamide gel in $1 \times \mathrm{TBE}$ at $400 \mathrm{~V}$ for $30 \mathrm{~min}$. RNA was transferred to a nylon membrane (Hybond+, GE Healthcare; Owl semi-dry electroblotter) in $1 \times$ TBE for $20 \mathrm{~min}$ at $2.5 \mathrm{~mA} / \mathrm{cm}^{2}$, which was then probed using a ${ }^{32} \mathrm{P}$-labeled probe complementary to the $3^{\prime}$ (Fig. 1B,C) or $5^{\prime}$ (Fig. 7B) portion of U4 (Table 2) and exposed to a phosphor screen.

Stability of wild-type and mutant U4 transcripts was tested in the presence of depleted splicing extract. We added 2.4 pmol IVT $\mathrm{U} 4$ to a $\mathrm{U} 4$ degradation reaction after the $30^{\circ} \mathrm{C}$ incubation, and it was incubated for $12 \mathrm{~min}$ at $23^{\circ} \mathrm{C}$. Then 4 fmol cold actin transcript was added and incubated for an additional $30 \mathrm{~min}$ at $23^{\circ} \mathrm{C}$ to simulate the conditions of a splicing reaction. The reactions were then treated as above, and the Northern blot was probed with the 5' U4 probe (Table 2). For control reactions, $200 \mu \mathrm{L}$ stop buffer was added to $2.4 \mathrm{pmol}$ of each IVT U4 in the absence of depleted splicing extract and processed as above. Stability was calculated as the percentage of $\mathrm{U} 4$ transcript remaining relative to the control.

Autoradiograms were visualized using a Cyclone Phosphorimager and OptiQuant software (Packard Instruments). Image contrast and brightness were linearly adjusted using Adobe Photoshop to enhance clarity.

\section{Pre-mRNA splicing assay}

To assess the effect of U4 degradation on splicing activity, 4 fmol internally ${ }^{32} \mathrm{P}$-GTP-labeled actin pre-mRNA in vitro transcript was added to the degradation reaction and incubated for $30 \mathrm{~min}$ at $23^{\circ} \mathrm{C}$. To assess reconstitution of splicing in U4-depleted extract by wild-type or mutant U4, 2.4 pmol IVT U4 (unless otherwise indicated) was added following the $\mathrm{U} 4$ depletion reaction and incubated for $12 \mathrm{~min}$ at $23^{\circ} \mathrm{C}$ to allow snRNP assembly to occur prior to addition of internally labeled actin pre-mRNA and a further incubation for $30 \mathrm{~min}$ at $23^{\circ} \mathrm{C}$. Splicing reactions were terminated by addition of $200 \mu \mathrm{L}$ stop buffer, extracted with phenol/chloroform, ethanol precipitated, and electrophoresed through a $6 \%(19: 1) 7 \mathrm{M}$ urea denaturing polyacrylamide gel for $1 \mathrm{~h}$ at $400 \mathrm{~V}$. The gel was then exposed to a phosphor screen at $-80^{\circ} \mathrm{C}$.

To calculate splicing efficiency, autoradiograms were visualized and quantified as described above. The percentage of splicing was calculated by dividing the intensity of bands corresponding to product (mRNA and lariat) by the total intensity of the starting material plus the product (pre-mRNA, lariat, and mRNA). The level of splicing activity restored by the U4 mutants was expressed as a percentage of that of wild-type $\mathrm{U} 4$ by subtracting the residual splicing activity found in the U4-depleted reaction from both the mutant and wild-type $\mathrm{U} 4$ activities and dividing the former value by the latter. Splicing efficiencies were determined at least in triplicate.

\section{Analysis of U4/U6 base-pairing}

Following the U4 degradation reaction, IVT U4 (2.4 pmol for single reactions, or up to $24 \mathrm{pmol}$ in titrations) was added, and the mixture was incubated for $12 \mathrm{~min}$ at $23^{\circ} \mathrm{C}$ to allow interaction with U6 to occur. Two hundred microliters of stop buffer was then added, and the samples were phenol/chloroform extracted and ethanol precipitated. To examine the effect of degradation of the U4 central domain on U4/U6 interaction, stop buffer was added immediately following the degradation reaction. Precipitated RNA was resuspended in $10 \mu \mathrm{L}$ hybridization buffer ( $150 \mathrm{mM} \mathrm{NaCl}, 50 \mathrm{mM}$ Tris- $\mathrm{HCl}$ at $\mathrm{pH} 7.4,1 \mathrm{mM}$ EDTA) and incubated for $15 \mathrm{~min}$ at $23^{\circ} \mathrm{C}$ with $\sim 200-500 \mathrm{fmol}(100,000 \mathrm{cpm})$ ${ }^{32}$ P-labeled U6-467 probe (Table 2). Samples were electrophoresed through a precooled $9 \%$ (29:1) non-denaturing polyacrylamide gel in $1 \times \mathrm{TBE}$ for $1 \mathrm{~h}$ at $4^{\circ} \mathrm{C}$ at $300 \mathrm{~V}$. The gel was exposed to a phosphor screen at $-80^{\circ} \mathrm{C}$.

To calculate base-pairing efficiency, autoradiograms were visualized and quantified as described above. Base-pairing efficiency was calculated by dividing the intensity of the U4/U6 band by the total intensity of both bands (U4/U6 and free U6). The level of base-pairing activity restored by the U4 mutants was expressed as a percentage of that of wild-type $\mathrm{U} 4$ by subtracting the residual base-pairing found in the U4-depleted reaction from both the mutant and wild-type $\mathrm{U} 4$ activities and dividing the former value by the latter. Base-pairing efficiencies were determined at least in triplicate.

\section{Calculation of apparent $K_{D}$ values for U4/U6 formation}

Absolute values for di-snRNP formation were plotted as a function of the concentration of added IVT U4 in Kaleidagraph (Synergy Software, version 4.1.2), and the data were fit to the curve U4/U6 $(\%)=\left(100 \times[\mathrm{U} 4]^{2}\right) /\left([\mathrm{U} 4]^{2}+\mathrm{K}_{\mathrm{D}}{ }^{2}\right)$. Values for the error and $\mathrm{R}^{2}$ were calculated in Kaleidagraph. As is frequently found in binding experiments, apparent $\mathrm{K}_{\mathrm{D}}$ fits were apparently cooperative, and allowing a free parameter for the Hill coefficient yielded a value close to 2. We therefore set the Hill coefficient to exactly 2 for our fits to minimize the number of free parameters. In addition, the apparent $K_{D}$ clearly results from several competing processes, including at least the rate of U4/U6 formation and the rate of Brr2-mediated U4/U6 unwinding. It should not be interpreted as a true equilibrium constant for U4/U6 formation. Nevertheless, 
the U4 titration data demonstrate that U4/U6 formation behaves as a simple equilibrium, which justifies using the apparent $K_{D}$ to compare the ability of $\mathrm{U} 4$ variants to base pair with $\mathrm{U} 6$.

\section{SnRNP analysis}

To assess the formation of tri-snRNP in depleted extracts reconstituted with U4, $1 \mathrm{mM}$ spermidine was added to the U4/U6 base-pairing reconstitution reaction. Protein/RNA complexes were electrophoresed through a precooled $4 \%$ (80:1) native polyacrylamide gel in $1 \times$ TGM buffer $(50 \mathrm{mM}$ Tris base, $50 \mathrm{mM}$ glycine, $2 \mathrm{mM} \mathrm{MgCl}_{2}$ ) for $3 \mathrm{~h}$ at $4^{\circ} \mathrm{C}$ at $160 \mathrm{~V}$ (Raghunathan and Guthrie 1998a). Gels were transferred to nylon membrane in $20 \mathrm{mM} \mathrm{NaPO}_{4}$ (pH 6.4), $20 \mathrm{~min}$ at $2.5 \mathrm{~mA} / \mathrm{cm}^{2}$, and then probed with ${ }^{32} \mathrm{P}$-labeled U6-6D (Table 2).

\section{ACKNOWLEDGMENTS}

We thank K. Aukema for technical assistance and H. Oh for initial experiments with loop mutants, as well as other members of the Rader laboratory and Rémy Bordonné for helpful discussions and comments on the manuscript. This work was supported by NSERC Discovery Grant 298521 to S.D.R., NSERC Canada Graduate Scholarship 347734, MSFHR Trainee Award 857(06-1)BM, two UNBC Health Research Project Awards, and a UNBC Research Project Award to A.J.H.

Received December 7, 2011; accepted January 26, 2012.

\section{REFERENCES}

Ansari A, Schwer B. 1995. SLU7 and a novel activity, SSF1, act during the PRP16-dependent step of yeast pre-mRNA splicing. EMBO J 14: 4001-4009.

Aukema KG, Chohan KK, Plourde GL, Reimer KB, Rader SD. 2009. Small molecule inhibitors of yeast pre-mRNA splicing. ACS Chem Biol 4: 759-768.

Ayadi L, Miller M, Banroques J. 1997. Mutations within the yeast U4/ U6 snRNP protein Prp4 affect a late stage of spliceosome assembly. RNA 3: 197-209.

Berget SM, Robberson BL. 1986. U1, U2, and U4/U6 small nuclear ribonucleoproteins are required for in vitro splicing but not polyadenylation. Cell 46: 691-696.

Bordonné R, Banroques J, Abelson J, Guthrie C. 1990. Domains of yeast U4 spliceosomal RNA required for PRP4 protein binding, snRNP-snRNP interactions, and pre-mRNA splicing in vivo. Genes Dev 4: 1185-1196.

Brenner TJ, Guthrie C. 2006. Assembly of Snu114 into U5 snRNP requires Prp8 and a functional GTPase domain. RNA 12: 862871.

Brow DA, Guthrie C. 1988. Spliceosomal RNA U6 is remarkably conserved from yeast to mammals. Nature 334: 213-218.

Cheng SC, Abelson J. 1987. Spliceosome assembly in yeast. Genes Dev 1: 1014-1027.

Fabrizio P, McPheeters DS, Abelson J. 1989. In vitro assembly of yeast U6 snRNP: a functional assay. Genes Dev 3: 2137-2150.

Ghetti A, Company M, Abelson J. 1995. Specificity of Prp24 binding to RNA: a role for Prp24 in the dynamic interaction of U4 and U6 snRNAs. RNA 1: 132-145.

Guthrie C, Patterson B. 1988. Spliceosomal snRNAs. Annu Rev Genet 22: $387-419$.

He H, Liyanarachchi S, Akagi K, Nagy R, Li J, Dietrich RC, Li W, Sebastian N, Wen B, Xin B, et al. 2011. Mutations in U4atac
snRNA, a component of the minor spliceosome, in the developmental disorder MOPD I. Science 332: 238-240.

Horowitz DS, Abelson J. 1993. A U5 small nuclear ribonucleoprotein particle protein involved only in the second step of premRNA splicing in Saccharomyces cerevisiae. Mol Cell Biol 13: 2959-2970.

Hu J, Field D, Wang A, Friesen JD. 1995a. The central region of the yeast U4 snRNA is not important for hammerhead catalysis, but may act as a domain spacer. Nucleic Acids Symp Ser 1995: 25-28.

$\mathrm{Hu}$ J, Xu D, Schappert K, Xu Y, Friesen JD. 1995b. Mutational analysis of Saccharomyces cerevisiae U4 small nuclear RNA identifies functionally important domains. Mol Cell Biol 15: 12741285.

Lamond AI, Konarska MM, Grabowski PJ, Sharp PA. 1988. Spliceosome assembly involves the binding and release of U4 small nuclear ribonucleoprotein. Proc Natl Acad Sci 85: 411-415.

Leung AKW, Nagai K, Li J. 2011. Structure of the spliceosomal U4 snRNP core domain and its implication for snRNP biogenesis. Nature 473: 536-539.

Mayas RM, Maita H, Staley JP. 2006. Exon ligation is proofread by the DExD/H-box ATPase Prp22p. Nat Struct Mol Biol 13: 482-490.

McGrail JC, O'Keefe RT. 2008. The U1, U2 and U5 snRNAs crosslink to the $5^{\prime}$ exon during yeast pre-mRNA splicing. Nucleic Acids Res 36: $814-825$.

McGrail JC, Tatum EM, O'Keefe RT. 2006. Mutation in the U2 snRNA influences exon interactions of U5 snRNA loop 1 during pre-mRNA splicing. EMBO J 25: 3813-3822.

McPheeters DS, Fabrizio P, Abelson J. 1989. In vitro reconstitution of functional yeast U2 snRNPs. Genes Dev 3: 2124-2136.

Myslinski E, Branlant C. 1991. A phylogenetic study of U4 snRNA reveals the existence of an evolutionarily conserved secondary structure corresponding to "free" U4 snRNA. Biochimie 73: 17-28.

O'Keefe RT, Norman C, Newman AJ. 1996. The invariant U5 snRNA loop 1 sequence is dispensable for the first catalytic step of premRNA splicing in yeast. Cell 86: 679-689.

Owczarzy R, Tataurov AV, Wu Y, Manthey JA, McQuisten KA, Almabrazi HG, Pedersen KF, Lin Y, Garretson J, McEntaggart NO, et al. 2008. IDT SciTools: a suite for analysis and design of nucleic acid oligomers. Nucleic Acids Res 36: W163-W169.

Raghunathan PL, Guthrie C. 1998a. A spliceosomal recycling factor that reanneals $\mathrm{U} 4$ and $\mathrm{U} 6$ small nuclear ribonucleoprotein particles. Science 279: 857-860.

Raghunathan PL, Guthrie C. 1998b. RNA unwinding in U4/U6 snRNPs requires ATP hydrolysis and the DEIH-box splicing factor Brr2. Curr Biol 8: 847-855.

Ségault V, Will CL, Sproat BS, Lührmann R. 1995. In vitro reconstitution of mammalian U2 and U5 snRNPs active in splicing: Sm proteins are functionally interchangeable and are essential for the formation of functional $\mathrm{U} 2$ and $\mathrm{U} 5$ snRNPs. EMBO $J$ 14: 4010-4021.

Shukla GC, Cole AJ, Dietrich RC, Padgett RA. 2002. Domains of human U4atac snRNA required for U12-dependent splicing in vivo. Nucleic Acids Res 30: 4650-4657.

Vankan P, McGuigan C, Mattaj IW. 1990. Domains of U4 and U6 snRNAs required for snRNP assembly and splicing complementation in Xenopus oocytes. EMBO J 9: 3397-3404.

Vankan P, McGuigan C, Mattaj IW. 1992. Roles of U4 and U6 snRNAs in the assembly of splicing complexes. EMBO J 11: 335343.

Vijayraghavan U, Parker R, Tamm J, Iimura Y, Rossi J, Abelson J, Guthrie C. 1986. Mutations in conserved intron sequences affect multiple steps in the yeast splicing pathway, particularly assembly of the spliceosome. EMBO J 5: 1683-1695.

Wersig C, Bindereif A. 1990. Conserved domains of human U4 snRNA required for snRNP and spliceosome assembly. Nucleic Acids Res 18: 6223-6229.

Wersig C, Bindereif A. 1992. Reconstitution of functional mammalian U4 small nuclear ribonucleoprotein: Sm protein binding is not essential for splicing in vitro. Mol Cell Biol 12: 1460-1468. 
Hayduk et al.

Wersig C, Guddat U, Pieler T, Bindereif A. 1992. Assembly and nuclear transport of the U4 and U4/U6 snRNPs. Exp Cell Res 199: 373-377.

Will CL, Rümpler S, Klein Gunnewiek J, van Venrooij WJ, Lührmann R. 1996. In vitro reconstitution of mammalian U1 snRNPs active in splicing: the U1-C protein enhances the formation of early (E) spliceosomal complexes. Nucleic Acids Res 24: 46144623.
Wolff T, Bindereif A. 1992. Reconstituted mammalian U4/U6 snRNP complements splicing: a mutational analysis. EMBO J 11: 345-359.

Xu Y, Petersen-Bjørn S, Friesen JD. 1990. The PRP4 (RNA4) protein of Saccharomyces cerevisiae is associated with the $5^{\prime}$ portion of the U4 small nuclear RNA. Mol Cell Biol 10: 1217-1225.

Yean SL, Lin RJ. 1991. U4 small nuclear RNA dissociates from a yeast spliceosome and does not participate in the subsequent splicing reaction. Mol Cell Biol 11: 5571-5577. 

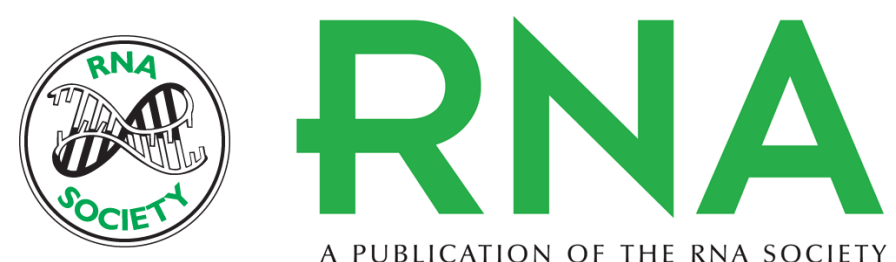

A PUBLICATION OF THE RNA SOCIETY

\section{In vitro reconstitution of yeast splicing with U4 snRNA reveals multiple roles for the 3 ' stem-loop}

Amy J. Hayduk, Martha R. Stark and Stephen D. Rader

RNA 2012 18: 1075-1090 originally published online March 12, 2012

Access the most recent version at doi:10.1261/rna.031757.111

$\begin{array}{ll}\text { References } & \begin{array}{l}\text { This article cites } 38 \text { articles, } 15 \text { of which can be accessed free at: } \\ \text { http://rnajournal.cshlp.org/content/18/5/1075.full.html\#ref-list-1 }\end{array}\end{array}$

License

Email Alerting Receive free email alerts when new articles cite this article - sign up in the box at the Service top right corner of the article or click here. 